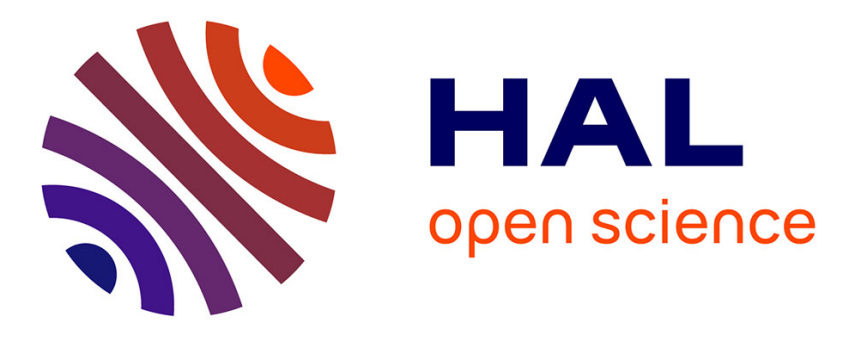

\title{
A control-oriented model of the current profile in Tokamak plasma
}

Emmanuel Witrant, Emmanuel Joffrin, Sylvain Brémond, G. Giruzzi, Didier Mazon, Oliviero Barana, Philippe Moreau

\section{- To cite this version:}

Emmanuel Witrant, Emmanuel Joffrin, Sylvain Brémond, G. Giruzzi, Didier Mazon, et al.. A controloriented model of the current profile in Tokamak plasma. Plasma Physics and Controlled Fusion, 2007, 49, pp.1075-1105. 10.1088/0741-3335/49/7/009 . hal-00387534

HAL Id: hal-00387534

https://hal.science/hal-00387534

Submitted on 26 May 2009

HAL is a multi-disciplinary open access archive for the deposit and dissemination of scientific research documents, whether they are published or not. The documents may come from teaching and research institutions in France or abroad, or from public or private research centers.
L'archive ouverte pluridisciplinaire HAL, est destinée au dépôt et à la diffusion de documents scientifiques de niveau recherche, publiés ou non, émanant des établissements d'enseignement et de recherche français ou étrangers, des laboratoires publics ou privés. 


\title{
A control-oriented model of the current profile in Tokamak plasma
}

\author{
E WITRANT ${ }^{1}$, E JOFFRIN ${ }^{2}$, S BRÉMOND $^{2}$, G GIRUZZI $^{2}$, D \\ MAZON $^{2}$, O BARANA ${ }^{3}$ and P MOREAU ${ }^{2}, 6$ June 2007. \\ ${ }^{1}$ Association EURATOM-VR, School of Electrical Engineering, Royal Institute of \\ Technology KTH, 10044 Stockholm, Sweden \\ 2 Association EURATOM-CEA, CEA/DSM/DRFC CEA-Cadarache, 13108 Saint Paul Lez \\ Durance, France \\ ${ }^{3}$ Consorzio RFX - Associazione EURATOM-ENEA sulla Fusione, Corso Stati Uniti 4, \\ I-35127 Padova, Italy
}

E-mail: emmanuel.witrant@ee.kth.se

\begin{abstract}
This paper proposes a control-oriented approach to the tokamak plasma current profile dynamics. It is established based on a consistent set of simplified relationships, in particular for the microwave current drive sources, rather than exact physical modelling. Assuming that a proper model for advanced control schemes can be established using the socalled cylindrical approximation and neglecting the diamagnetic effects, we propose a model that focuses on the flux diffusion (from which the current profile is inferred). Its inputs are some real-time measurements available on modern tokamaks and the effects of some major actuators, such as the magnetic coils, Lower Hybrid (LHCD), Electron and Ion Cyclotron Frequency (ECCD and ICRH) systems, are particularly taken into account. More precisely, the non-inductive current profile sources are modelled as 3-parameters functions of the control inputs derived either from approximate theoretical formulae for the ECCD and bootstrap terms or from experimental scaling laws specifically developed from Hard X-ray Tore Supra data for the LHCD influence. The use of scaling laws in this model reflects the fact that the operation of future reactors will certainly depend upon a great number of scaling laws and specific engineering parameters. The discretisation issues are also specifically addressed, to ensure the robustness with respect to discretisation errors and the efficiency (in terms of computation time) of the associated algorithm. This model is compared with experimental results and the CRONOS solver for Tore Supra Tokamak.
\end{abstract}

PACS numbers: 52.55.Fa

Submitted to: Plasma Phys. Control. Fusion 


\section{Introduction}

In the coming years the main challenge in the fusion community will be the development of experimental scenarios for ITER, the International Tokamak Experimental Reactor. Amongst them, the so-called advanced tokamak steady-state ones will play a significant role, since they will allow to reproduce and study (on a smaller scale), the conditions that are expected to be obtained in a fusion plant of reduced size and costs [1]. In these scenarios a particular emphasis is given to the current density profile and to the way of producing the plasma current $I_{P}$ : due to the intrinsic limited availability of magnetic flux in the fusion devices, needed to sustain a purely inductive current, $I_{P}$ will have to be mainly generated by non-inductive sources. In particular, the importance of the real-time safety factor profile ( $q$-profile) control is emphasized in [2], where an interesting overview on recent advances and key issues in real-time tokamak plasma control is provided. A Proportional-Integral (PI) feedback control strategy, based on a simple linear model, is also proposed and its efficiency is illustrated by experimental results, which motivate further research developments in this direction.

The control of so-called "advanced" plasma regimes $[1,3,4]$ for steady state high performance tokamak operation is a challenge, in particular because of the non-linear coupling between the current density and the pressure profiles. In a burning plasma, the alpha-particle power will also be a strong function of these profiles, and, through its effect on the bootstrap current, will be at the origin of a large (though ultra-slow) redistribution of the current density. The possible destabilization of adverse Toroidal Alfvén Eigenmodes (TAE) - such as the drift kinetic modes that are anticipated to appear at high values of the central safety factor [5] as well as potential thermal instabilities due to the ITB dynamics will further complicate the issue. This motivates the need for further investigation of plasma profiles shaping to guarantee and control steady-state operation of the plasmas.

As far as experiments are concerned, real-time control of the internal inductance parameter (a measure of the current profile shape) has been achieved with LHCD on Tore Supra [6]. Improvement of plasma performance through active modification of the current density and pressure profiles in advanced plasma regimes with ITB's, through heating and current drive, or by inducing sheared plasma rotation, has also been the goal of intense research for example on TFTR [7], JT-60U [8, 9, 10], DIII-D [11], Alcator C-Mod [12] and JET [13, 14, 15]. Regarding the real-time (closed loop) control issues, some experimental investigations were carried on JET, especially on the regulation of lumped parameters characterizing the pressure profile in ITB discharges [16], for fixed magnetic flux configuration, of the full safety factor profile during the ITB preforming phase $[17,18]$ for non-inductive steady-state regime (using a linear relationship between the $q$-profile and the actuators), and, more recently, of the full $q$-profile during the main heating phase of the discharges.

The former control approaches have shown the interest of appropriate control methods to improve the plasma performances. Nevertheless, they are based on identified linear models of the plasma and/or semi-empirical tuning of the gains of a proportional-integrator controller, rendering the real-time control particularly sensitive to the operating conditions. The aim 
of our work is then to propose a new, control-oriented model of the tokamak plasma that best reproduces the main influence of power and voltage modulations on the dynamics of the plasma profiles. This model does not pretend to have the accuracy of complex solvers (such as CRONOS, EFIT or EQUINOX), but has to represent the main control inputs influences on the plasma dynamics and to provide for some outputs of major interest for real-time control. It is then a first step towards model-based control of the plasma profile.

We focus on the flux diffusion dynamics and include some key physical knowledge on the tokamak plasma as well as the use of experimental results, which allows to represent the plasma behaviour for a large range of operating conditions and provides for a simplified and computationally efficient estimation of the main dynamics. Indeed, some approximate formulas are used to compute the resistivity and bootstrap current while the temperature and density profiles are estimated thanks to dedicated scaling laws. The non-inductive current profile sources are modelled as Gaussian distributions depending on the control inputs derived either from approximate theoretical formulas for ECCD or from experimental scaling laws specifically developed from Hard X-ray Tore Supra data for the LH term. The input-output relationships are detailed and special care is given to the discretisation issues. Such a model, essential for the phase of controller design (similarly to the numerous works done on plasma shape control), can then be used to quickly test some control laws for various operating conditions, to investigate the influence of specific parameters and to provide for a real time indication of not directly measurable quantities (such as the currents and $q$-factor profiles).

In order to keep the proposed plasma description as general as possible and to allow for advanced control methods, the model is presented as a non-linear system. Indeed, nonlinear control and stability analysis is a field on intense research that can be drawn back to the end of the $19^{\text {th }}$ century, with Lyapunov stability theory [19]. Numerous results have been obtained during the $20^{\text {th }}$ century, on stability analysis (i.e. absolute stability, passivity and small-gains theorems, or input-output stability) as well as constructive control approaches (i.e. backstepping, non-linear adaptive control, feedforwarding or non-linear model-predictive control). An interesting historical survey of these topics is presented in [20] and in [21], which proposes an overview of non-linear model-predictive control (one of the most widely used control method in industry). Nowadays, numerous textbooks are available, presenting nonlinear control from a global point of view [22, 23, 24] or focused on implementation issues $[25,26]$, differential geometric analysis $[27,28]$ or specific control methods $[29,30,31]$, to cite some of the main references in this field. Our aim is then to propose a general inputoutput simplified description that allows for different control strategies (including the nonlinear approaches) rather than focusing on a specific method.

This paper is organized as follows. First, the magnetic flux diffusion equation, with its initial and boundary conditions, is presented in Section 2. The estimation of the temperature and density profiles using some scaling laws based on experimental results is proposed in Section 3. Section 4 details the influence of the previous profiles on the flux diffusion, through the resistivity and the bootstrap current. The discretisation of the poloidal flux dynamics is investigated in Section 5, where an implicit-explicit discretisation scheme in time with a variable step in space is proposed. The inductive (magnetic coils) and non-inductive (ECCD 


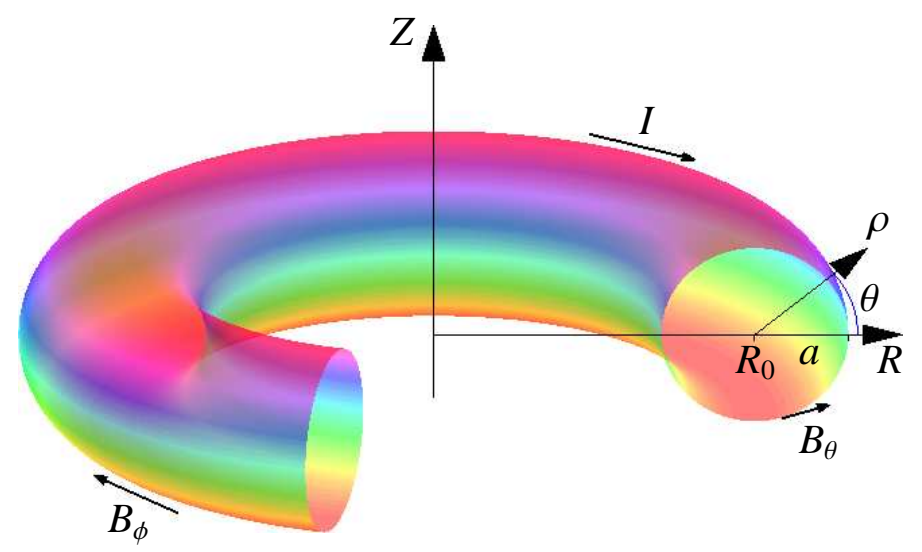

Figure 1. Plasma coordinates and sign convention.

and LHCD systems) inputs are described in Section 6. Section 7 details the computation of some model outputs of main interest. Finally, the numerical results obtained with the simulator associated with this model are compared with some experimental data from Tore Supra facility and with CRONOS code outputs in Section 8.

\section{Magnetic flux diffusion}

The notations and units of the main physical variables are summarized in Table 1 . The physical variable considered here is the flux $\psi(R, Z)$ of the magnetic field $B(R, Z)$ passing through a disc centred on the toroidal axis at height $Z$ and with a surface $S=\pi R^{2}$, where $R$ is the large plasma radius, as depicted in Figure 1. It is defined in flux per radian as

$$
\psi(R, Z) \doteq \frac{1}{2 \pi} \int_{S} \mathbf{B}(R, Z) \cdot d \mathbf{S}
$$

The dynamics of the poloidal flux is set by a diffusion equation, obtained from $[32,33]$ as

$$
\frac{\partial \psi}{\partial t}(\rho, t)=D(\rho, t) \frac{\partial^{2} \psi}{\partial \rho^{2}}+G(\rho, t) \frac{\partial \psi}{\partial \rho}+S(\rho, t)
$$

where $D(\rho, t)$ and $G(\rho, t)$ are transport coefficients, $S(\rho, t)$ is a source term and $\rho$ is the toroidal flux coefficient indexing the magnetic surfaces, defined as $\rho=\left(2 \phi / B_{\phi_{0}}\right)^{1 / 2}$, where $\phi(\rho, t)$ is the toroidal flux per radian and $B_{\phi_{0}}(t)$ is the central magnetic field. The transport coefficients and the source term are given by $\neq$

$$
D(\rho, t)=\frac{\eta_{/ /} C_{2}}{\mu_{0} C_{3}}, \quad G(\rho, t)=\frac{\eta_{/ /} \rho}{\mu_{0} C_{3}^{2}} \frac{\partial}{\partial \rho}\left(\frac{C_{2} C_{3}}{\rho}\right) \quad \text { and } \quad S(\rho, t)=\frac{\eta_{/ /} V^{\prime}}{F C_{3}}\left\langle\mathbf{j}_{n i} \cdot \mathbf{B}\right\rangle=\frac{\eta_{/ /} V^{\prime} B_{\phi_{0}}}{F C_{3}} j_{n i}
$$

where $\eta_{/ /}(\rho, t)$ is the resistivity, $\mu_{0}=4 \pi \times 10^{-7} \mathrm{H} / \mathrm{m}$ is the permeability of free space, $j_{n i}(\rho, t)$ is the non-inductive current source, including both the bootstrap effect and the microwave current drive, $F$ is the diamagnetic function, $V(\rho, t)$ is the plasma volume and $V^{\prime}=\partial V / \partial \rho$.

$¥$ In order to simplify the equations, the space and time dependencies are specified in the definitions of the variables and omitted otherwise. 
Furthermore,

$$
C_{2}=V^{\prime}\left\langle\frac{\|\rho\|^{2}}{R^{2}}\right\rangle, \quad C_{3}=V^{\prime}\left\langle\frac{1}{R^{2}}\right\rangle, \quad j_{n i}(\rho, t) \doteq \frac{<\mathbf{j}_{n i} \cdot \mathbf{B}>}{B_{\phi_{0}}} \quad \text { and } \quad\langle A\rangle \doteq \frac{\partial}{\partial V} \int_{V} A d V
$$

where $\langle A\rangle$ denotes the average of $A$ on the flux surface which contains $V$.

We suppose that the diamagnetic effect (due to the poloidal currents) can be neglected, which implies that $\rho$ can be considered as a geometric coefficient. We also assume that the so-called cylindrical approximation of the plasma geometry (large aspect ration) can be used as a basis to establish some control schemes using this model. The former approximation implies that $\rho<<R_{0}, V=2 \pi^{2} \rho^{2} R_{0}$ and $V^{\prime}=4 \pi^{2} \rho R_{0}$. Using both hypotheses, the transport coefficients can be computed with

$$
F \doteq \frac{\mu_{0} I_{\text {tor }}}{2 \pi} \approx R_{0} B_{\phi_{0}} \quad \text { and } \quad C_{2}=C_{3}=4 \pi^{2} \frac{\rho}{R_{0}}
$$

were $I_{t o r}$ is the toroidal coils current. Note that the cylindrical approximation could be relieved using some approximate expressions for $C_{2}, C_{3}$ and $V$ thanks to analytical formulas for shifted circles or numerical computations based on the $2 \mathrm{D}$ equilibrium description.

Remark 1 A detailed analysis of this model, and more particularly concerning the choice of the coordinates, is presented in [34]. Simulations of the diffusion equation expressed in cylindrical and toroidal coordinates (taking into account the Shafranov shift) are compared with some experimental results. This comparison is performed through the value of $q(1, t)$ and shows that the proposed model fits well with the experimental results (the cylindrical approximation leads to a steady-state error of $10 \%$ and the toroidal model has an error of $2 \%)$. Other results show that the geometrical coefficients $C_{2}$ and $C_{3}$ are identical for both approximations up to a normalized radius of 0.8 and differs when approaching the plasma edge to reach a difference of $15 \%$.

With the previous approximations, the dynamics (1) of $\psi$ simplifies to

$$
\frac{\partial \psi}{\partial t}(\rho, t)=\frac{\eta_{/ /}}{\mu_{0}} \frac{\partial^{2} \psi}{\partial \rho^{2}}+\frac{\eta_{/ /}}{\mu_{0} \rho} \frac{\partial \psi}{\partial \rho}+\eta_{/ /} R_{0} j_{n i}
$$

where $\psi, \eta_{\| /}$and $j_{n i}$ are both space (through $\rho$ ) and time dependent. The spatial index $\rho \in[0, a]$, where $a$ is the minor plasma radius corresponding to the Last Closed Magnetic Surface (LCMS, constant if the diamagnetic effect is neglected) depicted in Figure 1, can be replaced by the normalized variable $x=\rho / a$. The diffusion equation considered finally writes as

$$
\frac{\partial \psi}{\partial t}(x, t)=\frac{\eta_{/ /}(x, t)}{\mu_{0} a^{2}}\left(\frac{\partial^{2} \psi}{\partial x^{2}}+\frac{1}{x} \frac{\partial \psi}{\partial x}\right)+\eta_{/ /}(x, t) R_{0} j_{n i}(x, t) .
$$

The initial and boundary conditions of this equation are detailed in the next subsections. 


\begin{tabular}{|c|c|c|}
\hline$a$ & radius of the LCMS & $\mathrm{m}$ \\
\hline $\mathbf{B}_{\phi}$ & toroidal magnetic field & $\mathrm{T}$ \\
\hline $\mathbf{B}_{\theta}$ & poloidal magnetic field & $\mathrm{T}$ \\
\hline$B_{\phi_{0}}$ & toroidal magnetic field (at the centre of the plasma) & $\mathrm{T}$ \\
\hline$C_{R}$ & conductivity reduction due to electron-electron collisions & \\
\hline$c$ & speed of light in free space, $3 \times 10^{8}$ & $m / s$ \\
\hline$e$ & electron charge, $1.6022 \times 10^{-19}$ & $C$ \\
\hline$f_{t}$ & fraction of trapped particles in the banana regime & \\
\hline$F$ & diamagnetic function & $T \times m$ \\
\hline$I$ & plasma current at $x$ & $A$ \\
\hline$I_{p}$ & total plasma current & $A$ \\
\hline$I_{\text {tor }}$ & toroidal coils current & $A$ \\
\hline$j_{n i}$ & non inductive effective current density & $A / m^{2}$ \\
\hline $\ln \Lambda$ & Coulomb logarithm & \\
\hline$M$ & average ion mass & \\
\hline$m_{e}$ & electron mass, $9.1096 \times 10^{-31}$ & $k g$ \\
\hline$n_{e}$ & electron density profile & $m^{-3}$ \\
\hline $\bar{n}_{e}$ & electron line average density & $m^{-2}$ \\
\hline$N_{/ /}$ & parallel refraction index & \\
\hline$P$ & loss power & $W$ \\
\hline$P_{c d}$ & ECCD power & $W$ \\
\hline$P_{i c r h}$ & ICRH power & $W$ \\
\hline$P_{l h}$ & LH power & $W$ \\
\hline$P_{t o t}$ & total input power & $W$ \\
\hline$R$ & major plasma radius & $m$ \\
\hline$R_{0}$ & magnetic centre location & $m$ \\
\hline$T_{e}$ & temperature profile & $e V$ \\
\hline$x$ & normalized radius & \\
\hline $\bar{Z}$ & effective value of the plasma charge & $C$ \\
\hline$\alpha_{e}$ & electron thermal speed & $m / s$ \\
\hline$\alpha_{T i}$ & ratio of ion versus electron temperature & \\
\hline$\epsilon$ & inverse aspect ratio $\left(a / R_{0}\right)$ & \\
\hline$\epsilon_{0}$ & permittivity of free space, $8.854 \times 10^{-12}$ & $F / m$ \\
\hline$\eta_{/ /}$ & plasma resistivity & $\Omega \times m$ \\
\hline$\gamma_{i}$ & exponential peaking coefficient of the variable $i$ & \\
\hline$\kappa$ & elongation & \\
\hline$\mu_{0}$ & permeability of free space, $4 \pi \times 10^{-7}$ & $H / m$ \\
\hline$v_{* e}$ & electron collisionality parameter & \\
\hline$\phi$ & magnetic flux of the toroidal field & $T / m^{2}$ \\
\hline$\psi$ & magnetic flux of the poloidal field & $T / m^{2}$ \\
\hline$\tau_{e}$ & electron collision time & $s$ \\
\hline$\tau_{t h}$ & thermal energy confinement time & $s$ \\
\hline
\end{tabular}

Table 1. Most relevant physical variables and units 


\subsection{Initial value}

The initial value of the poloidal flux $\psi\left(x, t_{0}\right)$ is determined from the initial safety factor profile $q\left(x, t_{0}\right)$ as follows. Defining the toroidal flux $\phi(x, t)$ as the magnetic flux per radians (to be consistent with the definition of $\psi$ ) passing through a poloidal surface centered at $R_{0}$ and with radius $\rho$, we have that

$$
\phi(x, t) \doteq \frac{1}{2 \pi} \int_{S_{p o l}} \mathbf{B} \cdot d \mathbf{S}_{p o l}=-\frac{1}{2 \pi} \int_{S_{p o l}} B_{\phi} \cdot d S_{p o l} \approx-\frac{B_{\phi_{0}} a^{2} x^{2}}{2} .
$$

The safety factor is consequently defined as

$$
q(x, t) \doteq \frac{d \phi}{d \psi}=\frac{\partial \phi / \partial x}{\partial \psi / \partial x}=-\frac{B_{\phi_{0}} a^{2} x}{\partial \psi / \partial x}
$$

and, integrating $\partial \psi / \partial \rho$ in space at time $t_{0}$, we have that

$$
\psi\left(x, t_{0}\right)=a^{2} B_{\phi_{0}} \int_{x}^{1} \frac{r}{q\left(r, t_{0}\right)} d r+\psi\left(1, t_{0}\right) .
$$

The choice of the constant term is motivated by the fact that $\psi(1, t)$ can be measured on the LCMS and can also constitute a boundary condition. The initial safety factor profile is given by

$$
q\left(x, t_{0}\right)=\left(q\left(0, t_{0}\right)-q\left(1, t_{0}\right)\right)\left(1-x^{\gamma_{q}}\right)+q\left(1, t_{0}\right)
$$

where $q(1, t)$ is computed as follows. First, Ampere's law is introduced to compute the plasma current $I(x, t)$ and $q$-profile as

$$
I(x, t)=-\frac{2 \pi x}{\mu_{0} R_{0}} \frac{\partial \psi}{\partial x} \Rightarrow q(x, t)=\frac{2 \pi a^{2} x^{2} B_{\phi_{0}}}{\mu_{0} R_{0} I} .
$$

The safety factor on the LCMS is then $q(1, t)=\left(2 \pi a^{2} B_{\phi_{0}}\right) /\left(\mu_{0} R_{0} I_{p}\right)$ where $I_{p}(t) \doteq I(1, t)$ is the total plasma current.

For simulation purposes, an arbitrary initial safety factor profile can be chosen and the poloidal flux will converge to its actual value modulo a constant bias. The convergence property is inherited from the stability property of the diffusion equation and the bias is of minor importance since the variables of main interest ( $q$ and current profiles) depend on the flux gradient $\partial \psi / \partial x$. Further developments of this model for estimation purposes may use the real-time measurements of $\psi(1, t)$ to minimize the error between the modelled flux value on the LCMS and the real one (this is equivalent to design an observer for the flux profile that uses the measurements on the LCMS).

\subsection{Boundary conditions}

Specific boundary conditions have to be considered both at the plasma centre and on the LCMS. At the centre of the plasma, the spatial variation of the flux is zero:

$$
\frac{\partial \psi}{\partial x}(0, t)=0
$$

On the LCMS two exclusive conditions can be considered: 
- on the flux variation, from (4),

$$
\frac{\partial \psi}{\partial x}(1, t)=-\frac{R_{0} \mu_{0} I_{p}(t)}{2 \pi}
$$

- on the flux rate

$$
\frac{\partial \psi}{\partial t}(1, t)=V_{\text {loop }}(t)
$$

The last two boundary conditions are set by the tension applied to the coils, since a local control loop on the poloidal coil allows to set this tension according to the desired plasma current $I_{p}$ or the desired loop voltage $V_{\text {loop }}$. This will be detailed in subsection 6.1.

To summarize the main results of this section, under the hypotheses that

H1) the diamagnetic effect is neglected,

H2) the plasma is described in cylindrical coordinates (large aspect ratio approximation),

the dynamics of the poloidal flux is described by (2) with the initial condition (3), the central boundary condition (5) and the edge boundary condition (6) or (7).

\section{Temperature and density profiles}

A first, classical approach to compute the temperature profile is based on the diffusion equation (i.e. for the electron temperature $T_{e}(\rho, t)$ expressed in cylindrical coordinates [35])

$$
\frac{3}{2} \frac{\partial}{\partial t}\left[n_{e} T_{e}\right]=\frac{1}{\rho} \frac{\partial}{\partial \rho}\left(\rho n_{e} \chi_{e}(\rho, t) \frac{\partial T_{e}}{\partial \rho}\right)-\frac{3 n_{e} T_{e}}{2 \tau_{d}}+S_{T}(\rho, t)
$$

where $n_{e}(\rho, t)$ is the electron density, $\chi_{e}(\rho, t)$ is the electron thermal diffusivity, $\tau_{d}$ is a constant damping time modelling the losses and $S_{T}(\rho, t)$ is the source term. Denoting the electron pressure profile as $p_{e}(x, t)$, a simplified model can be set thanks to the relationship [36]

$$
\chi_{e}=\alpha_{B} \frac{T_{e}}{B_{\phi_{0}}} \frac{a \nabla p_{e}}{p_{e}} q^{2}
$$

with $\alpha_{B}=2.5 \times 10^{-4}$. The source term has an amplitude such that

$$
\int_{0}^{1} S_{T s}(x, t) d x=P_{s}
$$

where the $s$ subscript refers to the heating system considered (LHCD or ICRH) and $P_{s}$ is the associated power input.

Considering the high level of uncertainty induced by diffusion models, such as the one presented above, to compute the temperature profile, we choose to use the empirical model proposed in [37], based on some experimental measurements. This model is established for Tore Supra operating in L-Mode but could easily be extended to other tokamaks or operating conditions by following the guidelines of the identification algorithm with appropriate experimental data. The main idea is to first estimate the normalized (with respect to $T_{e}(0, t)$ ) temperature profile shape with a set of sigmoid functions (these functions are close to 1 when 
$x=0$ and close to 0 when $x=1$ ), which shape parameters are related to the tokamak global parameters with scaling laws. The thermal energy confinement is then introduced to compute the confinement time $\tau_{\text {th }}$ with a scaling law similar to ITERL-96P(th) [38]. The main advantage of this approach is to consider specifically the influence of LHCD and ICRH systems on the profile shape, as well as to provide for a simplified and fast computation method to estimate the global temperature behaviour. The resulting accuracy is sufficient for the proposed control-oriented model. Indeed, from a physical point of view, the dynamics of $\psi$ (on which this paper is focused) has a time constant that is one order of magnitude larger than the temperature one. This difference of time scales motivates the fact that the global energy and steady-state variations are more important than the temperature transient behaviour in the time-variation of $\psi$. For Tore Supra tokamak operating in L-mode, the temperature estimation is described as follows.

The electron temperature profile is estimated with a sigmoid function as

$$
T_{e}(x, t) \approx \frac{\alpha(t)}{1+e^{-\beta(t)(x-\gamma(t))}} A_{T e}(t)
$$

where the normalized shape of the profile $T_{e}(x, t) / T_{e}(0, t)$ is estimated with a sigmoid function defined by its amplitude $\alpha(t)$, dilatation $\beta(t)$ and translation (inflection point) $\gamma(t)$. The amplitude of the profile is $A_{T e}(t)$ and computed from the plasma thermal energy, as detailed below. The extra degree of freedom introduced in the time-variation of $\alpha$ (which would ideally be 1) is motivated by the fact that, for the computation of the resistive properties of the plasma, the minimization of the estimation error over the complete profile is more important than an accurate estimation of the central temperature.

The shape parameters are set with the switched model

$$
\{\alpha, \beta, \gamma\}= \begin{cases}\left\{\alpha_{l h}, \beta_{l h}, \gamma_{l h}\right\} & \text { if } P_{l h} \neq 0 \\ \left\{\alpha_{\omega}, \beta_{\omega}, \gamma_{\omega}\right\} & \text { else. }\end{cases}
$$

This model then distinguishes the LHCD heating effect from the ohmic and ICRH ones. Selecting the most significant terms, the shape parameters are related to the global and engineering parameters with

$$
\begin{aligned}
& \left\{\begin{array}{l}
\alpha_{l h}=e^{-0.87} I_{p}^{-0.43} B_{\phi_{0}}^{0.63} N_{/ /}^{0.25}\left(1+\frac{P_{\text {icrh }}}{P_{\text {tot }}}\right)^{0.15} \\
\beta_{l h}=-e^{3.88} I_{p}^{0.31} B_{\phi_{0}}^{-0.86} \bar{n}_{e}^{-0.39} N_{/ /}^{-1.15} \\
\gamma_{l h}=e^{1.77} I_{p}^{1.40} B_{\phi_{0}}^{-1.76} N_{/ /}^{-0.45}\left(1+\frac{P_{\text {icrh }}}{P_{\text {tot }}}\right)^{-0.54}
\end{array}\right. \\
& \left\{\begin{array}{l}
\alpha_{\omega}=e^{-0.37} I_{p}^{-0.46} B_{\phi_{0}}^{0.23} \bar{n}_{e}^{0.22} \\
\beta_{\omega}=-e^{1.92} I_{p}^{0.38} \bar{n}_{e}^{-0.33} \\
\gamma_{\omega}=e^{-0.15} I_{p}^{1.03} B_{\phi_{0}}^{-0.51}\left(1+\frac{P_{\text {icrh }}}{P_{\text {tot }}}\right)^{-0.46}
\end{array}\right.
\end{aligned}
$$


where $N_{/ /}(t)$ is the parallel refraction index, $P_{i c r h}(t)$ is the ICRH power, $P_{l h}(t)$ is the LHCD power, $P_{t o t}(t)$ is the total input power and $\bar{n}_{e}(t)$ is the electron line average density. The units of the parameters used in the scaling laws are $\left[s, M A, T, 10^{19} \mathrm{~m}^{-3}, M W\right]$ for [time, current, density, power]. Note that $N_{/ /}$and $P_{i c r h}$ have more effect on the profile shape associated with LHCD than $P_{l h}$, which still appears implicitly in $P_{t o t}$. Indeed, identifying the exponent parameters with either $\left(1+P_{i c r h} / P_{t o t}\right)$ or $\left(1+P_{l h} / P_{t o t}\right)$ in the scaling law leads to the conclusion that the first term gives the minimum cost function. This can be physically explained by the fact that the presence (or absence) of ICRH has more effect on the shape than some modulations in $P_{l h}$. A more accurate model could be obtained by distinguishing the case when LHCD is operated alone from the case when both LHCD and ICRH are used.

To determine the central temperature, the plasma thermal energy $W_{t h}(t)$ can be written as

$$
W_{t h}(t)=W_{e}(t)+W_{i}(t)=\frac{3 e}{2} \int_{V}\left(n_{e} T_{e}+n_{i} T_{i}\right) d V=\frac{3 e}{2} \int_{V}\left(1+\alpha_{T i} \alpha_{n i}\right) n_{e} T_{e} d V
$$

where $n_{i}(x, t) \approx \alpha_{n i}(t) n_{e}(x, t)$ is the ions density, $T_{i}(x, t) \approx \alpha_{T i}(t) T_{e}(x, t)$ is the ions temperature, and $W_{t h}(t)$ and $W_{e, i}(t)$ are the electrons and ions energies, respectively. The density ratio is given $\alpha_{n i}(t) \approx(7-\bar{Z}(t)) / 6$, where $\bar{Z}(t)$ is the effective plasma charge, averaged on the small plasma radius. The ratio of ion to electron temperature is established from measurements taken at the centre of the plasma and obtained from the scaling law

$$
\alpha_{T i}(t) \approx 1-0.31\left(\frac{I_{p}}{B_{\phi_{0}}}\right)^{-0.38} \bar{n}_{e}^{-0.90}\left(1+\frac{P_{i c r h}}{P_{t o t}}\right)^{-1.62}\left(1+\frac{P_{l h}}{P_{t o t}}\right)^{1.36} .
$$

The electrons density $n_{e}(x, t)$ is approximated with

$$
n_{e}(x, t) \approx \frac{\gamma_{n}+1}{\gamma_{n}}\left(1-x^{\gamma_{n}}\right) \bar{n}_{e}(t)
$$

where $\gamma_{n}$ is the density peaking. From the previous approximations and the cylindrical coordinates hypothesis, the temperature profile amplitude is related to $W_{\text {th }}$ thanks to the relationship $A_{T e}(t)=\mathcal{A}(t) W_{t h}(t)$ with

$$
\mathcal{A}(t) \doteq\left[6 \pi^{2} a^{2} R_{0} e\left(1+\alpha_{T i} \alpha_{n i}\right) \int_{0}^{1} n_{e}(x, t) x \frac{\alpha(t)}{1+e^{-\beta(t)(x-\gamma(t))}} d x\right]^{-1}
$$

and $W_{t h}$ is estimated with

$$
\left\{\begin{array}{l}
\tau_{t h}(t)=0.135 I_{p}^{0.94} B_{\phi_{0}}^{-0.15} \bar{n}_{e}^{0.78}\left(1+\frac{P_{l h}}{P_{t o t}}\right)^{0.13} P_{t o t}^{-0.78} \\
\frac{d W_{t h}}{d t}=P_{t o t}-\frac{1}{\tau_{t h}} W_{t h}, \quad W_{t h}(0)=P_{t o t}(0) \tau_{t h}(0)
\end{array}\right.
$$

where $\tau_{t h}(t)$ is the thermal energy confinement time with the scaling law established in [37]. The scaling law ITERL-96P(th) is more general (based on measurements from different tokamaks) and can also be used in this scheme. In that case, $\tau_{t h}$ is given by

$$
\tau_{\text {th,ITER }}=0.14 I_{p}^{0.96} B_{\phi_{0}}^{0.03} \bar{n}_{e}^{0.40} P_{\text {tot }}^{-0.73} .
$$


Remark 2 For a real-time use of this model, the temperature profile can also be accurately measured directly from the Electron Cyclotron Emission (ECE diagnostic) for $x=0-0.8$ and the density profile can be obtained from combined interferometers (these measurements were used to set the proposed scaling laws). In this case, the model can be used to provide, in real-time, for physical quantities that can not be measured directly, such as the safety factor, currents and current densities (associated with the different sources), and induced voltage profiles. The normalized inductance, confinement efficiency and Grad-Shafranov shift would also be available, as detailed in Section 7.

\section{Resistivity and bootstrap current}

The diffusion term in (2) is provided by $\eta_{/ /}$and the bootstrap current $j_{b s}(x, t)$ is an autogenerated source that introduces a non-linearity in the diffusion equation. Both of them introduce a coupling, which is varying in time and space, between the magnetic flux diffusion presented in Section 2 and the temperature and density profiles of Section 3.

\subsection{Resistivity model}

This parameter is computed using the results on neoclassical conductivity proposed in [39], where an approximate analytic approach is presented. First of all, the electron thermal velocity and Braginskii time are computed from the temperature and density profiles as [40]

$$
\alpha_{e}(x, t)=\sqrt{\frac{e T_{e}}{m_{e}}} \quad \text { and } \quad \tau_{e}(x, t)=\frac{12 \pi^{3 / 2} m_{e}^{1 / 2} \epsilon_{0}^{2}}{e^{5 / 2} \sqrt{2}} \frac{T_{e}^{3 / 2}}{n_{e} \ln \Lambda}
$$

where $e=1.6022 \times 10^{-19} \mathrm{C}$ is the electron charge, $\epsilon_{0}=8.854 \times 10^{-12} \mathrm{~F} / \mathrm{m}$ is the permittivity of free space, $m_{e}=9.1096 \times 10^{-31} \mathrm{~kg}$ is the electron mass and $\ln \Lambda(x, t)$ is the Coulomb logarithm, obtained from $\ln \Lambda(x, t)=31.318+\ln \left(T_{e} / \sqrt{n_{e}}\right)$. The parallel conductivity is then given by

$$
\frac{\sigma_{/ /}(x, t)}{\sigma_{0}}=\Lambda_{E}\left(1-\frac{f_{t}}{1+\xi v_{* e}}\right)\left(1-\frac{C_{R} f_{t}}{1+\xi v_{* e}}\right)
$$

with

$$
\begin{aligned}
& \sigma_{0}(x, t)=\frac{n_{e} e^{2}}{m_{e}} \tau_{e}, \quad \Lambda_{E}(\bar{Z})=\frac{3.40}{\bar{Z}}\left(\frac{1.13+\bar{Z}}{2.67+\bar{Z}}\right), \quad v_{* e}(x, t)=\frac{R_{0} q}{(x \epsilon)^{3 / 2} \alpha_{e} \tau_{e}}, \\
& f_{t}(x)=1-(1-x \epsilon)^{2}\left(1-(x \epsilon)^{2}\right)^{-1 / 2}(1+1.46 \sqrt{x \epsilon})^{-1}, \quad \xi(\bar{Z})=0.58+0.20 \bar{Z} \\
& \text { and } \quad C_{R}(\bar{Z})=\frac{0.56}{\bar{Z}}\left(\frac{3.0-\bar{Z}}{3.0+\bar{Z}}\right) .
\end{aligned}
$$

where $v_{* e}(x, t)$ is the electron collisionality parameter, $f_{t}(x)$ is the fraction of trapped particles in banana regime and $C_{R}(\bar{Z})$ is the conductivity reduction due to electron-electron collisions. The resistivity is finally inferred from $\sigma_{/ /}$as $\eta_{/ /}(x, t) \doteq 1 / \sigma_{/ /}$.

Remark 3 The validity of this approach is investigated in [41], where various models of conductivity (Hirsmann analytic formula, Hirshman formulation and Shaing formulation) are 
compared with experimental results. The model presented here (Hirshman analytic formula) is valid for low density, for arbitrary aspect ratio and banana regime (the collisionality goes to zero), or for finite collisionality and $\epsilon<<1$.

\subsection{Bootstrap current source}

This current is generated by trapped particles and may be the main source of non inductive current in specific scenarios (high bootstrap experiments). We present here the model derived by Hirshman [42] and presented in a comparative perspective in [43]. It is a single ion, collisionless regime model, which writes in our framework as

$$
\frac{<\mathbf{j} \cdot \mathbf{B}>_{b s}}{<\mathbf{B} \cdot \nabla \phi>}=\frac{p_{e}}{<1 / R^{2}>}\left\{A_{1}\left[\frac{1}{p_{e}} \frac{d p_{e}}{d \psi}+\frac{p_{i}}{p_{e}}\left(\frac{1}{p_{i}} \frac{d p_{i}}{d \psi}-\alpha_{i} \frac{1}{T_{i}} \frac{d T_{i}}{d \psi}\right)\right]-A_{2} \frac{1}{T_{e}} \frac{d T_{e}}{d \psi}\right\}
$$

where $p_{i}(x, t)$ is the pressure due to ions and

$$
\begin{aligned}
& A_{1}(x, t)=x_{t}\left[0.754+2.21 \bar{Z}+\bar{Z}^{2}+x_{t}\left(0.348+1.243 \bar{Z}+\bar{Z}^{2}\right)\right] / D_{e} \\
& A_{2}(x, t)=x_{t}(0.884+2.074 \bar{Z}) / D_{e}, \quad \alpha_{i}(x)=\frac{1.172}{1.0+0.462 x_{t}} \\
& D_{e}(x, t)=1.414 \bar{Z}+\bar{Z}^{2}+x_{t}\left(0.754+2.657 \bar{Z}+2 \bar{Z}^{2}\right)+x_{t}^{2}\left(0.348+1.243 \bar{Z}+\bar{Z}^{2}\right)
\end{aligned}
$$

where $x_{t}(x)$ is the ratio of trapped to circulating particles $f_{t} /\left(1-f_{t}\right)$. Considering the cylindrical coordinates approximation and defining the bootstrap current as

$$
j_{b s} \doteq \frac{<\mathbf{j} \cdot \mathbf{B}>_{b s}}{B_{\phi_{0}}}=\frac{1}{R_{0}} \frac{<\mathbf{j} \cdot \mathbf{B}>_{b s}}{<\mathbf{B} \cdot \nabla \phi>}
$$

we have

$$
j_{b s}(x, t)=\frac{p_{e} R_{0}}{\partial \psi / \partial x}\left\{A_{1}\left[\frac{1}{p_{e}} \frac{\partial p_{e}}{\partial x}+\frac{p_{i}}{p_{e}}\left(\frac{1}{p_{i}} \frac{\partial p_{i}}{\partial x}-\alpha_{i} \frac{1}{T_{i}} \frac{\partial T_{i}}{\partial x}\right)\right]-A_{2} \frac{1}{T_{e}} \frac{\partial T_{e}}{\partial x}\right\}
$$

The relationships $p_{e}=e n_{e} T_{e}$ and $p_{i}=e n_{i} T_{i}$ are introduced to express the bootstrap current in terms of temperature and density profiles as

$$
j_{b s}(x, t)=\frac{e R_{0}}{\partial \psi / \partial x}\left\{\left(A_{1}-A_{2}\right) n_{e} \frac{\partial T_{e}}{\partial x}+A_{1} T_{e} \frac{\partial n_{e}}{\partial x}+A_{1}\left(1-\alpha_{i}\right) n_{i} \frac{\partial T_{i}}{\partial x}+A_{1} T_{i} \frac{\partial n_{i}}{\partial x}\right\}
$$

Another possibility is to estimate the fraction of the total current due to the bootstrap effect with the fitting law proposed in [44].

\section{Discretisation of the poloidal flux dynamics}

Considering the dynamics obtained in (2), we wish to discretise

$$
\dot{\psi}(x, t)=\frac{\eta_{/ /}(x, t)}{\mu_{0} a^{2}}\left(\psi^{\prime \prime}(x, t)+\frac{1}{x} \psi^{\prime}(x, t)\right)+\eta_{/ /}(x, t) R_{0} j_{n i}(x, t)
$$

where $\dot{\psi} \doteq \partial \psi / \partial t$ and $\psi^{\prime} \doteq \partial \psi / \partial x$. Applying the spatial and temporal discretisation methods (A.1)-(A.3) described in Appendix A, the previous equation writes as (i.e. in the explicit case)

$$
\begin{aligned}
\dot{\psi}\left(x_{i}, t\right)_{e x} & =\frac{\eta_{/ / i, j}}{\mu_{0} a^{2}}\left(d_{2} \psi_{i+1, j}-d_{3} \psi_{i, j}+d_{4} \psi_{i-1, j}\right)+\frac{d_{1} \eta_{/ / i, j}}{\mu_{0} a^{2} x_{i}}\left(\psi_{i+1, j}-\psi_{i-1, j}\right)+\eta_{/ / i, j} R_{0} j_{n i i, j} \\
& =\eta_{/ / i, j}\left(e_{1} \psi_{i+1, j}-e_{2} \psi_{i, j}+e_{3} \psi_{i-1, j}+R_{0} j_{n i i, j}\right)
\end{aligned}
$$


with

$$
e_{1}(i)=\frac{d_{2}(i) x_{i}+d_{1}(i)}{\mu_{0} a^{2} x_{i}}, \quad e_{2}(i)=\frac{d_{3}(i)}{\mu_{0} a^{2}} \quad \text { and } \quad e_{3}(i)=\frac{d_{4}(i) x_{i}-d_{1}(i)}{\mu_{0} a^{2} x_{i}}
$$

where the subscript $i=1 \ldots N$ denotes the spatial discretisation points and $j$ refers to the time samples. Similarly, for the implicit case we have that

$$
\dot{\psi}\left(x_{i}, t\right)_{i m}=\eta_{/ / i, j+1}\left(e_{1} \psi_{i+1, j+1}-e_{2} \psi_{i, j+1}+e_{3} \psi_{i-1, j+1}+R_{0} j_{n i i, j}\right) .
$$

Note that we used $j_{n i i, j}$ instead of $j_{n i i, j+1}$ in the previous computation. This is motivated by the fact that $j_{n i i, j}$ is a non-linear function of $\psi_{1 \ldots N, j}$ (bootstrap effect and LH source when the boundary condition is set on $V_{\text {loop }}$ ). A formulation with $j_{n i i, j+1}$ would then prevent the use of a linear computation method that takes the sources at time $j$ as an input. Taking the time step sufficiently small compared to the system dynamics, this approximation introduces a negligible error that is worth the computation simplification and is compensated by the fact that the dynamics considered is stable.

Substituting the previous equalities into

$$
\left(\frac{\psi_{i, j+1}-\psi_{i, j}}{\delta t}\right)=h \dot{\psi}\left(x_{i}, t\right)_{e x}+(1-h) \dot{\psi}\left(x_{i}, t\right)_{i m}
$$

where $h \in[0,1]$ is the ratio of explicit to implicit time discretisation and $\delta t$ is the sampling time, a discretised version of (8) finally writes as

$$
\begin{aligned}
& A_{i, i+1, j} \psi_{i+1, j}+A_{i, i, j} \psi_{i, j}+A_{i, i-1, j} \psi_{i-1, j} \\
& -B_{i, i+1, j} \psi_{i+1, j+1}-B_{i, i, j} \psi_{i, j+1}-B_{i, i-1, j} \psi_{i-1, j+1}+S_{i, j}=0
\end{aligned}
$$

where $A_{j}$ and $B_{j}$ are time-varying $N \times N$ matrices. The notations $A_{i, k, j}$ and $B_{i, k, j}$ are introduced to denote the values of the matrix elements $(i, k)$ at the time sample $j$, and

$$
\begin{aligned}
& A_{i, i-1, j}=\eta_{/ / i, j} e_{3} h \delta t, A_{i, i, j}=1-\eta_{/ / i, j} e_{2} h \delta t \\
& A_{i, i+1, j}=\eta_{/ / i, j} e_{1} h \delta t, B_{i, i-1, j}=-\eta_{/ / i, j+1} e_{3}(1-h) \delta t \\
& B_{i, i, j}=1+\eta_{/ / i, j+1} e_{2}(1-h) \delta t, B_{i, i+1, j}=-\eta_{/ / i, j+1} e_{1}(1-h) \delta t \\
& S_{i, j}=R_{0} \delta t\left[h \eta_{/ / i, j}+(1-h) \eta_{/ / i, j+1}\right] j_{n i i, j}
\end{aligned}
$$

for $i=2, \ldots, N-1$ (the values at 1 and $N$ are given by the boundary conditions) and where $\delta t$ is the sampling time. Writing the poloidal flux and the sources term in the vector form

$$
\psi_{j}=\left[\begin{array}{lllll}
\psi_{1, j} & \psi_{2, j} & \ldots & \psi_{N, j}
\end{array}\right]^{T} \text { and } S_{j}=\left[\begin{array}{llll}
S_{1, j} & S_{2, j} & \ldots & S_{N, j}
\end{array}\right]^{T}
$$

(9) can be expressed, equivalently, in the matrix form

$$
A_{j} \psi_{j}-B_{j} \psi_{j+1}+S_{j}=0 \quad \Leftrightarrow \quad \psi_{j+1}=B_{j}^{-1} A_{j} \psi_{j}+B_{j}^{-1} S_{j} .
$$

Note that the matrix $B_{j}$ is tridiagonal, by construction. An appropriate inversion method such as the one proposed in [45] is therefore recommended to compute $B_{j}^{-1}$ with reduced computational cost.

The boundary conditions presented in subsection 2.2 may induce some numerical instability due to the discretisation scheme. Some appropriate specific discretisation methods are described bellow. 
At the centre: the proposed discretisation method leads to an ill defined central value of the flux dynamics when $x_{1}=0$ since

$$
\frac{\psi^{\prime}\left(x_{1}, t\right)}{x_{1}} \rightarrow \frac{0}{0}
$$

Consequently, we use the fact that the diffusion term close to the origin can be approximated by

$$
\frac{1}{x} \frac{\partial}{\partial x}\left[x \frac{\partial \psi}{\partial x}\right] \approx \frac{1}{\delta x_{2} / 4} \frac{\left(\delta x_{2} / 2\right) \times \psi_{1 / 2}^{\prime}}{\delta x_{2} / 2}=\frac{4}{\delta x_{2}} \psi_{1 / 2}^{\prime} \approx \frac{4}{\delta x_{2}^{2}}\left(\psi_{2}-\psi_{1}\right)
$$

with $\delta x_{i}=x_{i}-x_{i-1}$ to set the central dynamics as

$$
\dot{\psi}\left(x_{1}, t\right) \approx \eta_{/ / 1}(t)\left[\frac{4}{\mu_{0} a^{2} \delta x_{2}^{2}}\left(\psi_{2}-\psi_{1}\right)+R_{0} j_{n i 1}(t)\right] .
$$

The central terms of (9) are then computed with

$$
\begin{aligned}
& A_{1,2, j}=e_{b c 0} h \eta_{/ / 1, j}, \quad A_{1,1, j}=1-e_{b c 0} h \eta_{/ / 1, j}, \quad B_{1,2, j}=-e_{b c 0}(1-h) \eta_{/ / 1, j+1}, \\
& B_{1,1, j}=1+e_{b c 0}(1-h) \eta_{/ / 1, j+1} \quad \text { and } \quad S_{1, j}=\delta_{t} R_{0}\left(h \eta_{/ / 1, j} j_{n i 1, j}+(1-h) \eta_{/ / 1, j+1} j_{n i 1, j+1}\right) \\
& \text { where } e_{b c 0}=4 \delta_{t} /\left(\mu_{0} a^{2} \delta x_{2}^{2}\right) .
\end{aligned}
$$

At the edge, with $\psi^{\prime}(1, t)$ : the constraint on the flux variation $\psi^{\prime}(1, t)=u(t)$ implies that

$$
\frac{\psi_{N, j+1}-\psi_{N-1, j+1}}{\delta x_{N}}=u_{j+1} \quad \Leftrightarrow \quad-\frac{\psi_{N, j+1}}{\delta x_{N}}+\frac{\psi_{N-1, j+1}}{\delta x_{N}}+u_{j+1}=0 .
$$

The corresponding matrix coefficients are then

$$
A_{N, N-1, j}=A_{N, N, j}=0, \quad B_{N, N-1, j}=-B_{N, N, j}=-\frac{1}{\delta x_{N}} \quad \text { and } \quad S_{N, j}=u_{j+1} .
$$

The computation of $u(t)$ is directly obtained from (6) as $u(t)=-R_{0} \mu_{0} I_{p} /(2 \pi)$.

At the edge, with $\dot{\psi}(1, t)$ a last possibility to set the edge boundary condition is to use $\dot{\psi}(1, t)=V_{\text {loop }}(t)$. In this case we have

$$
\psi_{N, j+1}-\psi_{N, j}-\delta t V_{l o o p, j}=0
$$

and the matrix coefficients are

$A_{N-1, N, j}=B_{N-1, N, j}=A_{N, N-1, j}=B_{N, N-1, j}=0, \quad A_{N, N, j}=B_{N, N, j}=1 \quad$ and $\quad S_{N, j}=\delta t V_{\text {loop }, j}$.

\section{Model inputs}

The model inputs considered in this work are the boundary conditions at the plasma edge $\left(\psi^{\prime}(1, t)\right.$ or $\dot{\psi}(1, t)$, see subsection 2.2$)$ and the non inductive currents generated by the LHCD and ECCD systems. Both systems current deposits can be roughly modelled with Gaussian curves, described in Appendix B, whose shape depends on the global plasma parameters and power inputs. 


\subsection{Inductive current input}

The magnetic flux at the boundary $\dot{\psi}(1, t)$ is set by the coils surrounding the plasma and constitutes the inductive current input. This can be described by the classical transformer model where the coils generate the primary circuit while the plasma is the secondary, modelled as a single filament. The dynamics of the coils current $I_{c}(t)$ is then set by [46]

$$
\begin{aligned}
& L_{c} \dot{I}_{c}+M \dot{I}_{p}+R_{c} I_{c}-V_{c}=0 \\
& M \dot{I}_{c}+L_{p} \dot{I}_{p}+R_{p}\left(I_{p}-I_{N I}\right)=0
\end{aligned}
$$

where $R_{c}$ and $L_{c}$ are the coils resistance and internal inductance, $R_{p}$ and $L_{p}$ are the plasma resistance and inductance, $M$ is the matrix of mutual inductances, $V_{c}$ is the input voltage applied to the coils and $I_{N I}$ is the current generated by the non inductive sources. Note that the values of $R_{c}$ and $L_{c}$ are given from the coil properties while $M$ is obtained thanks to an equilibrium code (i.e. CEDRES on Tore Supra). The magnetic flux at the plasma boundary close to the coils $\psi_{a c}$ is then obtained with $\dot{\psi}_{a c}=M \dot{I}_{c}$. Considering the effects of the plasma current and inductance variations, the loop voltage $V_{\text {loop }}$ is obtained from [47]

$$
V_{\text {loop }}(t)=\dot{\psi}(1, t)=-\frac{1}{I_{p}} \frac{\partial}{\partial t}\left[\frac{L_{p} I_{p}^{2}}{2}\right]+\dot{\psi}_{a c}
$$

where $L_{p}=\mu_{0} R_{0} l_{i} / 2$ and $l_{i}$ is the normalized internal inductance. In practice, a local control law is set on the poloidal coils to adjust the value of $V_{c}$ according to a desired value of $V_{\text {loop }}$, which can be measured with a Rogowski coil. If the reference is set on the plasma current $I_{p}$ instead, then $V_{c}$ is such that the coils provide for the current necessary to complement the non inductive sources.

\subsection{ECCD deposit}

The total EC current is the sum of several deposits due to several EC beams, which parameters are denoted by the subscript $m$. Each current deposit is determined by the position of the steering mirror $\left(R_{a n t, m}, Z_{a n t, m}\right)$, its orientation in the poloidal and toroidal plane $\left(\phi_{p o l, m}, \phi_{t o r, m}\right)$, and the emission power $P_{c d, m}$, as presented in Figure 2. The results presented in this section are derived from [48] and [49]. The assumption is that the wave is absorbed by the plasma (and generates current) for

$$
R_{c}>R>R_{m}^{*}
$$

where

$$
R_{c}=n_{h} \frac{e \mu_{0} n_{b} n_{t}}{4 \pi^{2} m_{e}} \frac{I_{\text {ind }}}{f} \quad \text { and } \quad R_{m}^{*}=\frac{R_{\text {ant }, m}}{\sqrt{2}\left|\sin \phi_{\text {tor }, m}\right|}\left[1-\sqrt{1-\frac{4 R_{c}^{2}}{R_{\text {ant }, m}^{2}} \sin ^{2} \phi_{\text {tor }, m}}\right]^{1 / 2}
$$

with $n_{h}=1,2$ the harmonic considered, $n_{b}$ the number of toroidal coils, $n_{t}$ the number of wire loops per coil, $f$ the antenna frequency (in $G H z$ ) and $I_{\text {ind }}$ the coil induction current. For high magnetic field (O-mode) the first harmonic is absorbed $\left(n_{h}=1\right)$ while for low magnetic field 


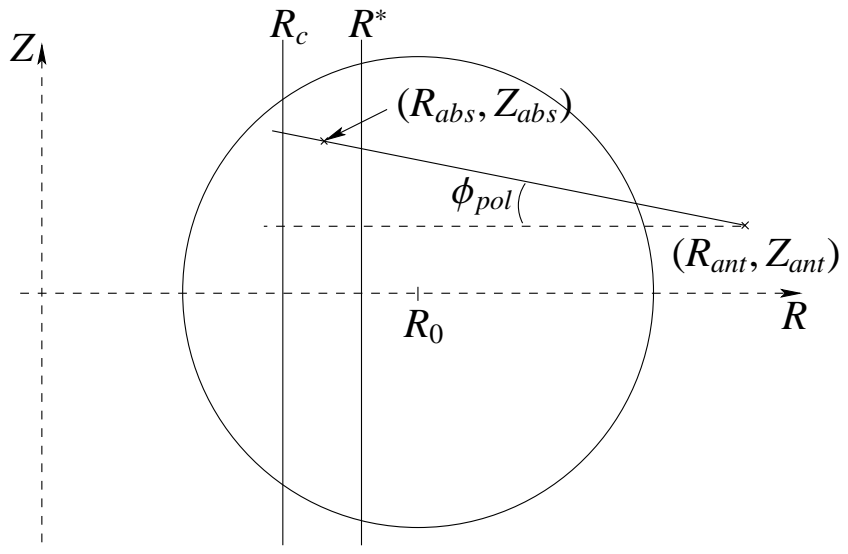

Figure 2. Poloidal deposit of the ECCD antenna (one mirror).

(X-mode) the deposit is generated by the second harmonic. The maximum is considered to happen at $R_{a b s, m}=\left(R_{c}+R_{m}^{*}\right) / 2$ and the $Z$ coordinate of each point is computed from

$$
Z_{m}(R)=Z_{a n t, m}+\left(R_{a n t, m}-R\right) \tan \phi_{p o l, m}
$$

The flux coordinates of the deposit are then obtained from

$$
\rho_{m}(R)=\left|\frac{R-R_{0}}{Z_{a n t, m}+\left(R_{a n t, m}-R\right) \tan \phi_{p o l, m}}\right| .
$$

The global efficiency factor $\gamma_{c d, m}(t)$ is computed as

$$
\gamma_{c d, m}(t)=\frac{\Gamma_{1} T_{e}\left(x_{a b s, m}\right)}{T_{e}\left(x_{a b s, m}\right)+10^{5}}\left\{1-\Gamma_{2}\left[\frac{\rho_{m}\left(R_{a b s, m}\right)+R_{a b s, m}-R_{0}}{R_{a b s, m}}\right]^{\Gamma_{3}}\right\}
$$

where

$$
x_{a b s, m}=\rho_{m} \frac{R_{a b s, m}}{a}, \quad \Gamma_{1}=\frac{6}{5+\bar{Z}}, \quad \Gamma_{2}=\frac{4(2+\bar{Z})}{3(1+\bar{Z})} \quad \text { and } \quad \Gamma_{3}=\frac{5+\bar{Z}}{2(1+\bar{Z})}
$$

and the amplitude of the current deposit $I_{c d, m}(t)$ is obtained from

$\gamma_{c d, m}=\frac{I_{c d, m}}{P_{c d, m}} R_{o} \bar{n}_{e} \times 10^{-20} \Leftrightarrow I_{c d, m}=\frac{\gamma_{c d, m} P_{c d, m}}{R_{o} \bar{n}_{e} \times 10^{-20}}=2 \pi a^{2} \int_{0}^{1} x j_{c d, m}(x, t) d x$

where $j_{c d, m}(x, t)$ is the current density induced by ECCD.

The next step is to express the coefficients of the Gaussian fitting curve

$$
j_{c d, m}=\vartheta_{c d, m} e^{-\left(\mu_{c d, m}-x\right)^{2} / 2 \sigma_{c d, m}}
$$

in terms of the engineering parameters derived previously. The mean and the variance are obtained as

$$
\mu_{c d, m}=\frac{\rho_{m}\left(R_{a b s, m}\right)}{a} \quad \text { and } \quad \sigma_{c d, m}=-\frac{\left(\mu_{c d, m}-\rho_{m}\left(R_{c}\right) / a\right)^{2}}{2 \ln \beta}
$$


where $\beta$ is chosen such that $j_{c d, m}\left(x_{c}\right)=\vartheta_{c d, m} \beta \approx 0$ (i.e. $\beta=1 / 10$, which is equivalent to set the Gaussian curve close to zero at $R_{c}$ ). The maximum value of the current deposit is consequently computed as

$$
\vartheta_{c d, m}=\frac{\gamma_{c d, m} P_{c d, m}}{R_{o} \bar{n}_{e}}\left[2 \pi a^{2} \int_{0}^{1} x e^{-\left(\mu_{c d, m}-x\right)^{2} / 2 \sigma_{c d, m}} d x\right]^{-1} .
$$

Finally, the ECCD source is positive if the antenna is directed against the plasma current $I_{p}$ (which means that it emits in the same direction as the plasma electrons) and the total current density profile induced by ECCD is

$$
j_{c d}(x, t)=\sum_{m=1}^{6} j_{c d, m}(x, t) \times \operatorname{sign}\left(\phi_{t o r, m}\right) .
$$

Example 1 Tore Supra tokamak ECCD system is currently working with two beams. The current deposit is set with

$$
\max \left(P_{c d}\right)=\left[\begin{array}{ll}
250 & 300
\end{array}\right] \times 10^{3}, \quad R_{\text {ant }}=\left[\begin{array}{lll}
3.53 & 3.53
\end{array}\right] \text { and } Z_{\text {ant }}=\left[\begin{array}{ll}
0 & 0.2
\end{array}\right] .
$$

Furthermore, $I_{\text {ind }}=137 \times B_{\phi_{0}} R_{0}, n_{B}=18$ and $n_{t}=2028$.

\subsection{LHCD deposit}

The current profile generated by this system cannot be adequately described with an analytical formula as it is the case for ECCD. Indeed, it strongly depends on the operating conditions and current density profile [50]. A more realistic way to estimate this profile is to use the emission of suprathermal electrons provided by the Hard X-Ray (HXR) measurements [51] to build up a scaling law from engineering control parameters. A classical guess is to suppose that the LH power deposit corresponds to the emission of electrons with an energy ranging from 60 to $80 \mathrm{keV}$, which is measured with the HXR diagnostic. It is motivated by the fact that LHCD system specifically generates a population of electron in this range of energies. Neglecting the thermal effect, we then consider that the radial Hard X-Ray emission profile corresponds to the current density profile $j_{l h}(x, t)$ [50].

The first step is to determine the shape of the current deposit from the HXR measurements. This can be done empirically from the global parameters thanks to the curve fitting approach described in Appendix C, where the width $w_{h x r}(t)$ and centre of the deposit $\mu_{h x r}(t)$ are estimated as

$$
\begin{aligned}
& \hat{w}_{h x r}(t)=0.53 B_{\phi_{0}}^{-0.24} I_{p}^{0.57} \bar{n}^{-0.08} P_{L H}^{0.13} N_{/ /}^{0.39} \\
& \hat{\mu}_{h x r}(t)=0.20 B_{\phi_{0}}^{-0.39} I_{p}^{0.71} \bar{n}^{-0.02} P_{L H}^{0.13} N_{/ /}^{1.20}
\end{aligned}
$$

where $N_{/ /}$is the parallel refractive index that can be computed from phase difference measurements. Note that a direct measurement of the parameters involved in the scaling law is available in real-time for advanced control schemes.

The total current deposit $I_{l h}(t)$ is computed from the plasma and LHCD parameters thanks to the current drive efficiency $\eta_{l h}(t)$ with the fitting laws proposed in [52] (for Tore Supra and 


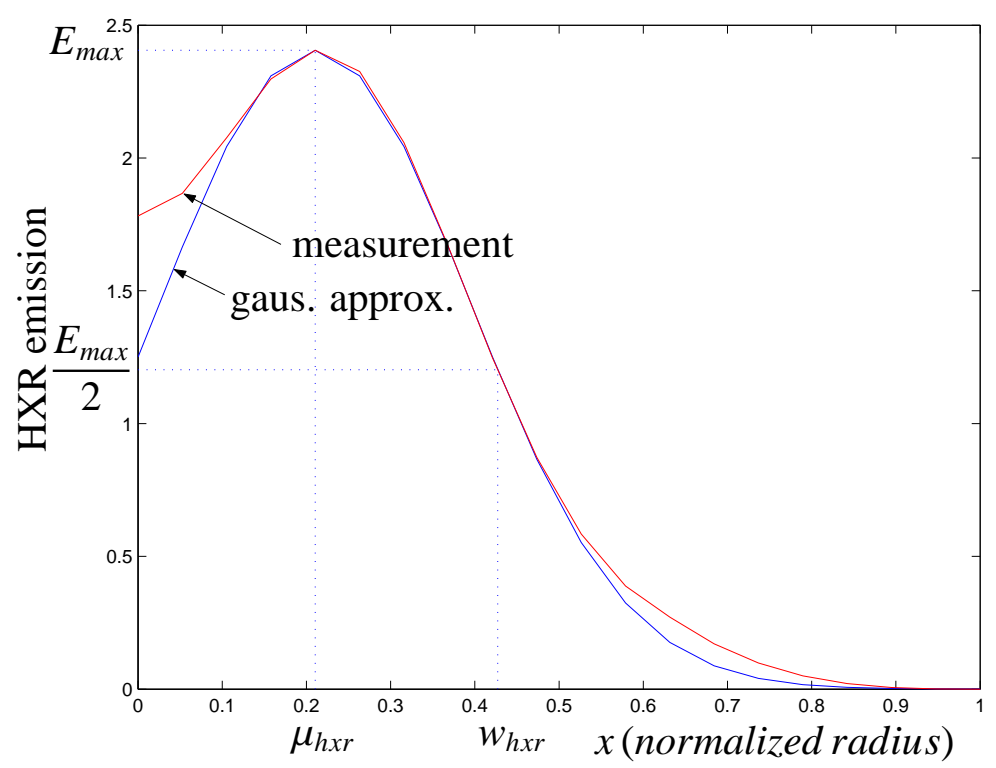

Figure 3. Measured LH deposit vs. its Gaussian approximation.

JET)

$$
\eta_{l h}(t)=3.39 D_{n}^{0.26} \tau_{t h}^{0.46} \bar{Z}^{-0.13} \text { or } \quad \eta_{l h}(t)=1.18 D_{n}^{0.55} I_{p}^{0.43} \bar{Z}^{-0.24}
$$

where $D_{n}(t) \approx 2.03-0.63 N_{/ /}$is the normalized directivity. The total current is then obtained as $I_{l h}(t)=\eta_{l h} P_{l h} /\left(\bar{n}_{e} R_{0}\right)$. The LHCD profile $j_{l h}(x, t)$ is finally described by the Gaussian approximation

$$
j_{l h}(x, t)=\vartheta_{l h} e^{-\left(\mu_{h x r}-x\right)^{2} / 2 \sigma_{l h}}
$$

with

$$
\sigma_{l h}(t)=\frac{\left(\mu_{h x r}-w_{h x r}\right)^{2}}{2 \ln 2} \quad \text { and } \quad \vartheta_{l h}(t)=I_{l h}\left[2 \pi a^{2} \int_{0}^{1} x e^{-\left(\mu_{h x r}-x\right)^{2} / 2 \sigma_{l h}} d x\right]^{-1} .
$$

Note that the integral term can be computed using numerical integration techniques or with the relationship

$$
\int_{0}^{1} x e^{-\left(\mu_{h x r}-x\right)^{2} / 2 \sigma_{l h}} d x=-\sigma\left(e^{-(1-\mu)^{2} / 2 \sigma}-e^{-\mu^{2} / 2 \sigma}\right)+\mu \sqrt{\frac{\sigma \pi}{2}}\left[\operatorname{erf}\left(\frac{1-\mu}{\sqrt{2 \sigma}}\right)-\operatorname{erf}\left(\frac{-\mu}{\sqrt{2 \sigma}}\right)\right]
$$

A comparison between the measured and estimated HXR emission profile is presented on Figure 3, where $E_{\max }$ is the maximum emission value, for a typical LHCD pulse. The uncertainty on the measured values close to the origin is high, which further supports the choice of a Gaussian curve to fit the measurements.

\subsection{Resulting model properties}

To summarize the results obtained in the previous sections, the proposed model is mainly based on a physical analysis of the plasma flux diffusion and current sources. This analysis is completed with some scaling laws to compute the LHCD current deposit, and possibly the temperature profiles. The shape of LHCD deposit is based on experimental measurements carried on Tore Supra while its amplitude is established from both Tore Supra 
and JET measurements. For control design purposes, the temperature profiles can be roughly approximated thanks to the proposed scaling laws or an analytical model. Another possibility, which should be preferred for advanced control design since it fully uses the available diagnostics, is to consider the temperature profiles as some real-time inputs to the model, provided by ECE.

The use of scaling laws, even if they imply that the model is not fully predictive, is motivated by the fact that a large amount of experimental data is available for the actual tokamaks and their use in the design of future facilities, as illustrated in [38]. The large amount of physical properties included in the model allows reducing the error and operation dependency induced by such laws to a level that is acceptable for control design purposes, where global dependencies are more important then detailed models.

Such an approach is also motivated by the results obtained in [18] and similar works, where encouraging closed-loop performances for the $q$-profile control were obtained using a simple linearised model identified from experimental data. Even if linear approaches structurally imply a strong dependency on the operating conditions, the previous results have shown the interest of model-based control design to regulate the plasma profiles. Our aim is then to contribute to further progresses in this direction by providing for a model with strong physical dependencies and not restricted to the linear framework, that still allows for real-time implementation and can be used in non-linear control schemes.

\section{Model outputs}

Several outputs of major interest for control applications are presented in this section, as well as a practical method to compensate for the cylindrical approximation and estimate the security factor and current densities profiles in toroidal coordinates.

\subsection{Total current and effective current density}

For an arbitrary current denoted with the subscript $s$ to indicate the source (ohmic, LHCD, ECCD or effective), the total current $I_{s}(x, t)$ is obtained, with the cylindrical approximation, by integrating the current density $j_{s}(x, t)$ on the surface $\pi a^{2} x^{2}$ as

$$
I_{s}(x, t)=2 \pi a^{2} \int_{0}^{x} x j_{s}(x, t) d x
$$

The effective current density of the plasma $j_{\phi}(x, t)$ is obtained from the spacial derivative of the previous equality and Ampere's law (4) as

$$
j_{\phi}(x, t)=\frac{1}{2 \pi a^{2} x} \frac{\partial I}{\partial x}=\frac{1}{2 \pi a^{2} x} \frac{\partial}{\partial x}\left[-\frac{2 \pi x}{\mu_{0} R_{0}} \frac{\partial \psi}{\partial x}\right]=-\frac{1}{\mu_{0} R_{0} a^{2} x} \frac{\partial}{\partial x}\left[x \frac{\partial \psi}{\partial x}\right] .
$$

Note that the toroidal current at the plasma edge $j_{\phi}(1, t)$ can be used to estimate the model precision since it is usually measured using a continuous Rogowski coil or a discrete set of magnetic coils surrounding the plasma. 


\subsection{Toroidal induced voltage}

It is induced by changes in both the primary circuit current and plasma current, and writes as $V(x, t)=\partial \psi / \partial t$. It is computed using the results of Section 5 as

$$
\begin{aligned}
V_{i, j}= & h \eta_{/ / i, j}\left(e_{1} \psi_{i+1, j}-e_{2} \psi_{i, j}+e_{3} \psi_{i-1, j}+R_{0} j_{n i i, j}\right) \\
& +(1-h) \eta_{/ / i, j+1}\left(e_{1} \psi_{i+1, j+1}-e_{2} \psi_{i, j+1}+e_{3} \psi_{i-1, j+1}+R_{0} j_{n i i, j+1}\right)
\end{aligned}
$$

for $i=2 \ldots N-1$ and with the appropriate boundary conditions for $V_{1, j}$ and $V_{N, j}$. Note that the voltage at the edge of the plasma $V(1, t)$ is the loop voltage $V_{\text {loop }}(t)$, which is measured with a toroidal loop of wire parallel to the plasma.

\subsection{Ohmic current}

This current is usefull to analyse the various components of the total current profile and is computed from

$$
<\mathbf{j} \cdot \mathbf{B}>_{\Omega}=\sigma_{/ /}<\mathbf{E} \cdot \mathbf{B}>=\sigma_{/ /} E_{/ /} B_{\phi_{0}}
$$

where $E(x, t)$ is the electric field. Introducing the voltage profile, we obtain, equivalently,

$$
j_{\Omega}(x, t)=\frac{<\mathbf{j} \cdot \mathbf{B}>_{\Omega}}{B_{\phi_{0}}}=-\frac{\sigma_{/ /}}{R} V(x, t) \approx-\frac{\sigma_{/ /}}{R_{0}} V(x, t) .
$$

\subsection{Normalized internal inductance}

Defined as [40]

$$
l_{i}(x, t) \doteq \frac{\bar{B}_{\theta}^{2}(x, t)}{B_{\theta}^{2}(1)}=\frac{2 \int_{0}^{1} B_{\theta}^{2}(x, t) x d x}{B_{\theta}^{2}(1)}
$$

it is expressed is terms of the poloidal flux using Biot-Savart law $B_{\theta}(x, t)=\psi^{\prime} /\left(a R_{0}\right)$ as

$$
l_{i}(x, t)=\frac{2 \int_{0}^{1} \psi^{\prime 2}(x, t) x d x}{\psi^{\prime 2}(1, t)}=\frac{8 \pi^{2}}{\mu_{0}^{2} R_{0}^{2} I_{p}^{2}} \int_{0}^{1} \psi^{\prime 2}(x, t) x d x
$$

\subsection{Confinement efficiency}

This is a global parameter that evaluates the confinement of the plasma pressure by the magnetic field. It is defined as the ratio between the average perpendicular pressure and the edge poloidal magnetic pressure [53, 40]

$$
\beta_{\theta}(t) \doteq \frac{<p>}{B_{\theta}^{2}(1, t) / 2 \mu_{0}}=\frac{4}{\mu_{0} R_{0} I_{p}^{2}} \int_{V} p d V=\frac{8 W_{t h}}{3 \mu_{0} R_{0} I_{p}^{2}}
$$

where the last equality is obtained from the cylindrical approximation. 


\subsection{Grad-Shafranov (GS) shift and geometrical correction}

An alternative to the computation of the GS equilibrium is to approximate the GS shift $\Delta(x, t)$ from the global parameters defined above. This allows for a comparison between the profiles obtained with the cylindrical approximation and those obtained in toroidal coordinates. The central value of the GS shift $\Delta(0, t)$ is approximated with

$$
\Delta(0, t) \approx \frac{a^{2}}{2 R_{0}}\left(\beta_{\theta}+\frac{l i}{2}\right)\left(1-\frac{a}{R_{0}}\right)
$$

Assigning a profile shape to the GS shift, it is computed as $\Delta(x, t) \approx \Delta(0, t)\left(1-x^{2}\right)$.

The GS shift can be used to estimate the $q$-profile in toroidal coordinates $q_{t o r}(x, t)$. Indeed, $q_{\text {tor }}$ is given by [34]

$$
q_{t o r}(\rho, t)=-\frac{B_{\phi_{0}} R_{0}}{\partial \psi / \partial x}\left[\Delta^{\prime}+\frac{\rho-\left(R_{0}+\Delta\right) \Delta^{\prime}}{\sqrt{\left(R_{0}+\Delta\right)^{2}-\rho^{2}}}\right] .
$$

The toroidal approximation is then obtained thanks to the relationship

$$
q_{\text {tor }}(x, t)=\frac{R_{0}}{a x}\left[\Delta^{\prime}+\frac{a x-\left(R_{0}+\Delta\right) \Delta^{\prime}}{\sqrt{\left(R_{0}+\Delta\right)^{2}-(a x)^{2}}}\right] \times q(x, t)
$$

where $q(x, t)$ is the safety factor profile considered previously and computed from the dynamics of $\psi$.

The effective plasma current density is more difficult to express in toroidal coordinates with a GS shift. Indeed, this would require to compute

$$
<\mathbf{j}_{\phi} \cdot \mathbf{B}_{\phi}>=-\frac{F C_{2 t o r}}{\mu_{0} V_{\text {tor }}^{\prime}}\left(\frac{\partial^{2} \psi}{\partial \rho^{2}}+\frac{\partial \ln \left(C_{2 t o r}\right)}{\partial \rho} \frac{\partial \psi}{\partial \rho}\right)
$$

with

$$
C_{2 \text { tor }}=4 \pi^{2}\left(\frac{\rho}{\sqrt{\left(R_{0}+\Delta\right)^{2}-\rho^{2}}}-\frac{\Delta^{\prime}}{\sqrt{1-\Delta^{\prime 2}}}\right) \frac{\rho}{\rho-\left(R_{0}+\Delta\right) \Delta^{\prime}}
$$

and $V_{\text {tor }}^{\prime}=4 \pi^{2} \rho\left(R_{0}+\Delta+\rho \Delta^{\prime} / 2\right)$. A simpler way to take into account the geometry issues is to use the definition of the effective current $I_{\phi}(x, t)=V^{\prime}<B_{\phi}^{2}>/ 2 \pi \mu_{0}$ and the ratio $I_{\phi t o r}(1, t) / I_{\phi c y l}(1, t)=1-\Delta_{0}(t) / 2$ to set the boundary condition (6) as $\psi^{\prime}(1, t)=$ $-R_{0} \mu_{0} I_{p}(t) / 2 \pi \times\left(1-\Delta_{0}(t) / 2\right)$.

\section{Simulations and comparisons with experimental results}

The control-oriented model derived in this paper is now compared with some experimental measurements and the results obtained with the CRONOS code [54], an integrated modelling tool that solves the transport equations along with the Grad-Shafranov equilibrium in toroidal coordinates. The complexity of this solver prevents any real-time implementation but it is an experimentally-based reference that we can use to validate our results. We refer to our model results with $\psi_{\text {sim }}$, as name and subscript for the associated simulator, and compare them with the experimental (exp) and CRONOS signals. 
This comparison does not intend to be exhaustive but focuses on two specific cases of main interest. The first one is an ohmic shot with ICRH modulations and illustrates the diffusive behaviour of the plasma model as well as some discretisation issues. The second one is a LHCD shot, to illustrate the impact of the proposed scaling laws related to this system and of a non-inductive current source. In both cases the boundary condition of the diffusion equation is set with the plasma current $I_{p}(t)$ and a test case with $V_{\text {loop }}(t)$ illustrates the LHCD shot. The simulations are carried with 20 uniformly distributed space steps and with a sampling time of $10 \mathrm{~ms}$.

\subsection{Ohmic and bootstrap effects}

We analyse here the model results on Tore Supra shot 33632, which doesn't involve ECCD nor LHCD. In this shot, the temperature and density profiles are modulated with the ICRH power and the plasma has the following steady-state characteristics: $I_{p}=1.0 \mathrm{MA}, B_{\phi_{0}}=3.19 \mathrm{~T}$ and $n_{e 0}=4.5-3.0 \times 10^{-19} \mathrm{~m}^{-3}$.
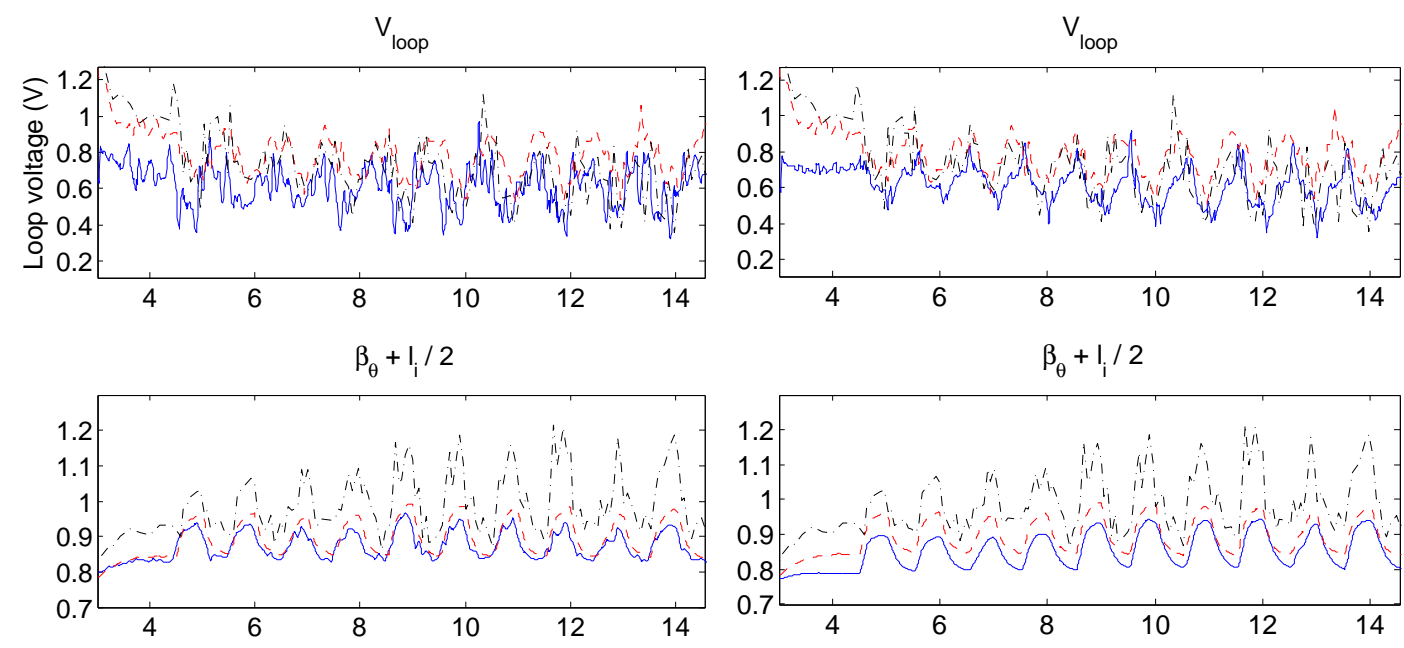

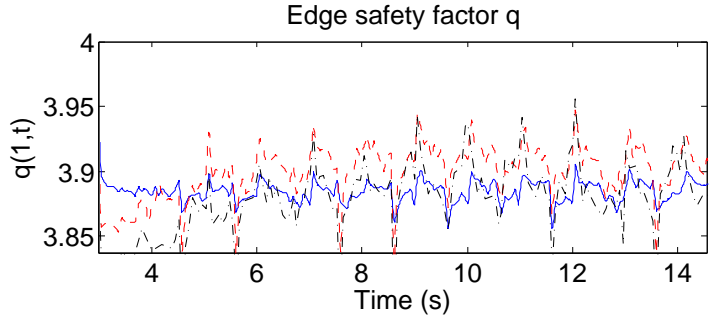

(a) With measured $T_{e}$ profile

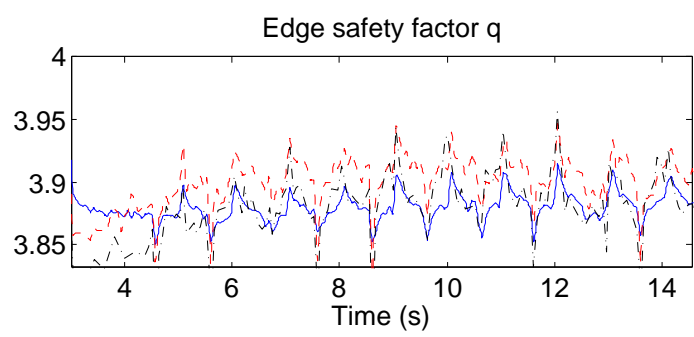

(b) With estimated $T_{e}$ profile

Figure 4. Comparison of $\psi_{\text {sim }}(-)$ with experimental measurements (--) and CRONOS signals (- - ) for shot TS-33632, based on the loop voltage (top), the measurement of $\beta_{\theta}+l_{i} / 2$ (middle) and the safety factor at the edge of the plasma (bottom).

The first comparison is performed on the magnetic measurement and presented in Figure 4, where the top part depicts the loop voltage, the middle part is $\beta_{\theta}+l_{i} / 2$ and the bottom part is the safety factor at the edge of the plasma $q_{t o r}(1, t)$ (computed with the corrected 
formula (10)). $\psi_{\text {sim }}$ is run both using the temperature and density profiles measurements (Figure 4(a)) and in a fully predictive way (Figure 4(b)). The later case means that $\psi_{\text {sim }}$ has only the global parameters of the plasma and $P_{i c r h}$ as inputs but doesn't use the temperature and density measurements. The proposed shape scaling laws and ITERL-96P(th) (for the thermal confinement time) set the temperature and density profiles. This test case is a worstcase example since the central safety factor is less than one and there is a high frequency saw tooth effect on the temperature profiles, which is not explicitly taken into account in the temperature model.

The results of $\psi_{\text {sim }}$ and CRONOS are equivalent for $V_{\text {loop }}$, except during the current ramp up and ramp down (not represented here) phases, which imply some particular phenomena that we didn't consider. The magnetic measurement of $\beta_{\theta}+l_{i} / 2$ is best represented with $\psi_{\text {sim }}$, even in the predictive mode. The constant bias on $q(1, t)$ due to the cylindrical approximation (reported in [34]) is successfully compensated by the geometrical correction proposed in Subsection 7.6.
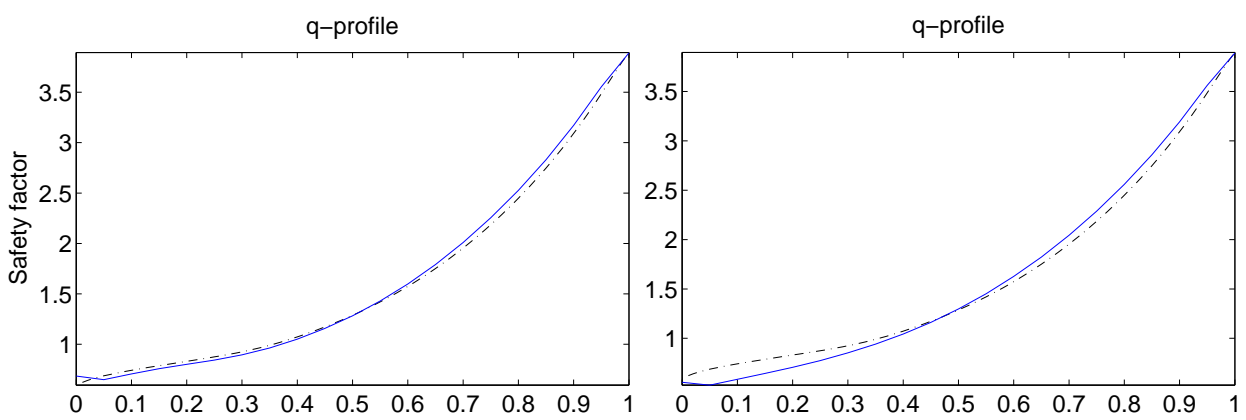

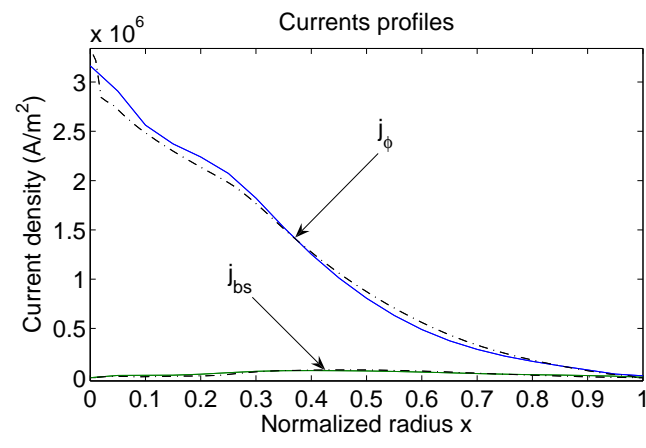

(a) With measured $T_{e}$ profile

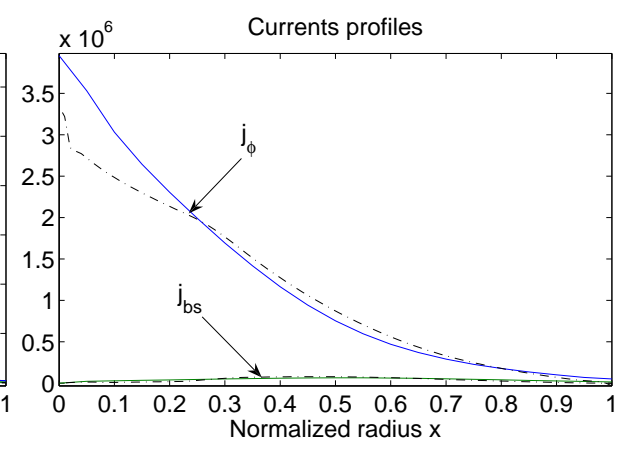

(b) With estimated $T_{e}$ profile

Figure 5. Comparison of $\psi_{\text {sim }}(-)$ with CRONOS (- - -) for shot TS-33632 at $t=7 \mathrm{~s}$, based on the safety factor profiles (top) and the current densities (effective $j_{\phi}$ and bootstrap $j_{b s}$ ) profiles (bottom).

The second comparison is focused on the safety factor and current densities profiles. There is no experimental measurements available for these physical variables so we compare $\psi_{\text {sim }}$ with CRONOS only. The difference between the use of the temperature and density profiles, and the use of their approximation is also illustrated and is more significant than in the first comparison. The $q$-profile is presented on top of Figure 5: we have an almost perfect matching between CRONOS and $\psi_{\text {sim }}$ when the measured profiles are used (Figure 5(a)) and 


\begin{tabular}{|l|l|c|c|c|}
\hline Mode & Discretisation & $\mathrm{N}$ & $e_{V_{\text {loop }}}(\%)$ & Simulation time (s) \\
\hline Meas. prof. & Uniform & 20 & 7.37 & 1.09 \\
Predictive & Uniform & 100 & 5.56 & 8.53 \\
Predictive & Non-uniform & 100 & 5.46 & 8.81 \\
Predictive & Uniform & 20 & 5.84 & 1.77 \\
Predictive & Non-uniform & 20 & 5.18 & 1.76 \\
Predictive & Uniform & 10 & 5.64 & 1.30 \\
Predictive & Non-uniform & 10 & 4.66 & 1.27 \\
\hline
\end{tabular}

Table 2. Effect of the spatial distribution

a small difference, located at the centre of the plasma, for the predictive mode (Figure 5(b)). The same conclusion is verified for the effective current density profile, with a more important mismatching at the centre. This illustrates the influence of the temperature on the flux diffusion, through $\eta_{/ /}$(which is proportional the $T_{e}^{3 / 2}$ ). Nevertheless, the accuracy of the $q$ profile may be sufficient for most control applications, especially considering that this specific case study includes saw teeth and that the model can be updated with real-time measurements from the Hard X-rays in advanced control setups.

The impact of the spatial discretisation strategy is presented in Table 2. The non-uniform distribution $\delta x_{i}=2 / N+\sqrt{a i}$, where $a$ is such that $\sum \delta x_{i}=1$, is compared with the uniform distribution for different numbers of discretisation points $N$ thanks to the resulting error on the loop voltage

$$
e_{V_{\text {loop }}} \doteq \frac{\int_{t_{0}}^{t_{f}}\left(V_{\text {loop- }-\psi_{\text {sim }}}(t)-V_{\text {loop-exp }}(t)\right)^{2} d t}{\int_{t_{0}}^{t_{f}} V_{\text {loop-exp }}^{2}(t) d t}
$$

and to the simulation time (obtained when the simulator is built with Matlab ${ }^{\circledR}$ on an Intel ${ }^{\circledR}$ $2 \mathrm{CPU}-2 \mathrm{GHz}$ PC operated with Windows $\mathrm{NT}^{\circledR}$ ). In each case, the non-uniform distribution is performing better than the uniform one in terms of the error on $V_{\text {loop }}$, and increasing the number of points does not necessarily decrease the error. This issue would clearly deserve further analysis, as the optimal discretisation would depend on the system properties and dynamics, but will not be investigated in this work. Instead, we proposed a discretisation scheme that allows for various possibilities and refer to $[55,56]$, where the effects of PDE models discretisation is thoroughly investigated.

\subsection{Lower Hybrid effect}

The LH system is now introduced in $\psi_{\text {sim }}$ and compared with CRONOS and experimental results. The simulator behaviour is tested for the operating conditions of Tore Supra shot 35109 , which is characterized by some variations in $N_{/ /}$, a constant $I_{p}(0.6 \mathrm{MA})$ and a constant power input (1.8 MW).

The magnetic measurements $V_{\text {loop }}(t), \beta_{\theta}+l_{i} / 2$ and $q(1, t)$ are presented in Figure 6 , where experimental measurements, CRONOS and $\psi_{\text {sim }}$ are compared. The cases with 

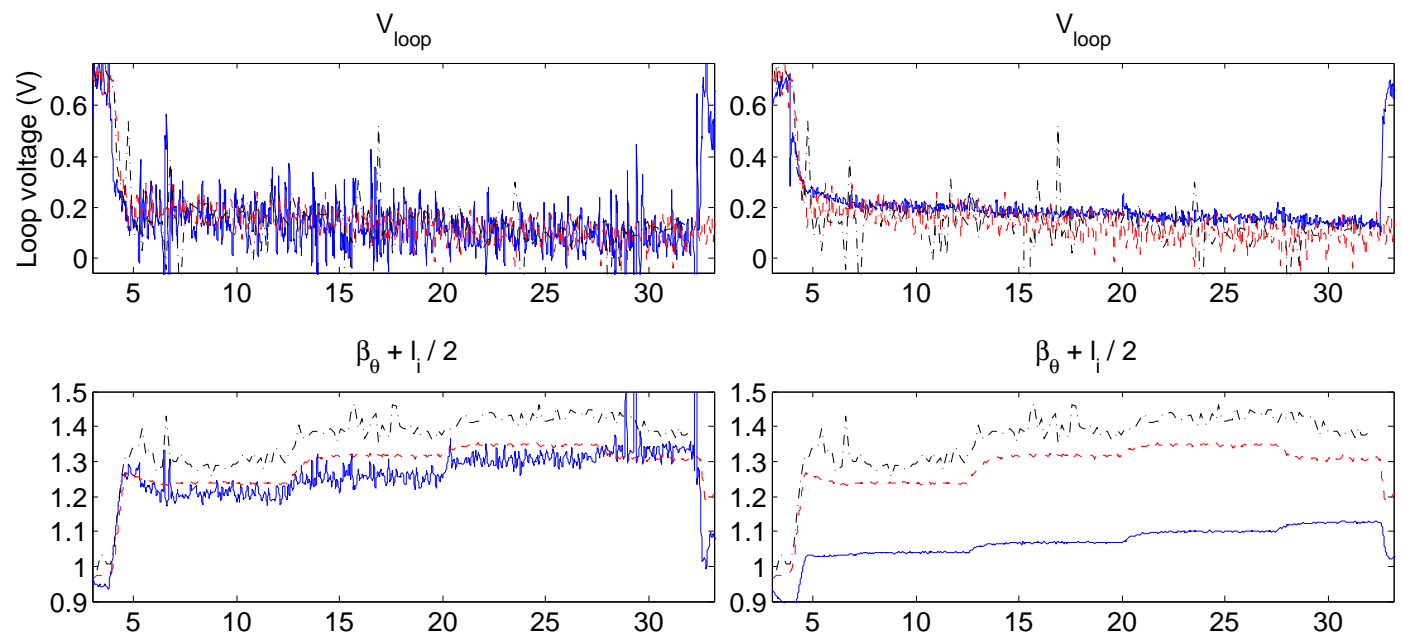

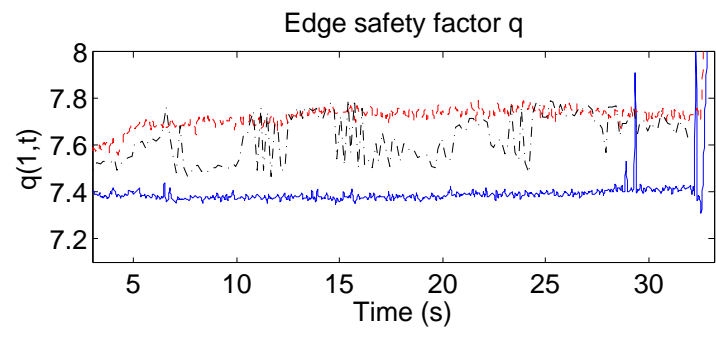

(a) With measured $T_{e}$ profile

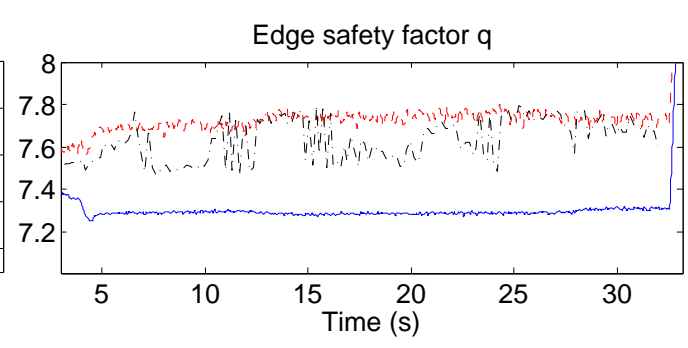

(b) With estimated $T_{e}$ profile

Figure 6. Comparison of $\psi_{\text {sim }}(-)$ with experimental measurements (--) and CRONOS signals (- - $)$ for shot TS-35109, based on the loop voltage (top), the measurement of $\beta_{\theta}+l_{i} / 2$ (middle) and the safety factor at the edge of the plasma (bottom).

measured and estimated temperature profiles are both considered, in Figures 6(a) and 6(b), respectively. In both cases there is no significant difference on $V_{\text {loop }}(t)$ while $q(1, t)$ is slightly underestimated (the correction proposed in Subsection 7.6 only partially compensate the cylindrical approximation effect). The main difference appears on $\beta_{\theta}+l_{i} / 2$, which is underestimated when the temperature profile is estimated.

The safety factor and current densities profiles for $\psi_{\text {sim }}$ (run with measured and estimated temperature profiles) and CRONOS are presented in Figure 7. The $q$-profile provided by $\psi_{\text {sim }}$ has a small difference with CRONOS close to the centre, especially on Figure 7(b). The peculiar behaviour of CRONOS current profiles for $x<0.5$ may be due to some computational artefacts and should not be taken into account into this comparison. The profile of $j_{l h}$ is reasonably well represented as well as the bootstrap profile, which is particularly accurate when the measured temperatures and densities are available.

The last profiles, presented on Figure 8 , are obtained using $V_{\text {loop }}(t)$ to set the boundary condition. While these profiles are not as close to CRONOS results as those obtained with $I_{p}(t)$, they are still consistent with the plasma behaviour. The difference between the two cases $\left(I_{p}\right.$ and $V_{\text {loop }}$ ) mainly comes from the fact that the scaling laws are set with $I_{p}$, which means that the computation error induced on the plasma current when it is considered as an output is fed back into the model through the inputs and more specially in the predictive mode, as 

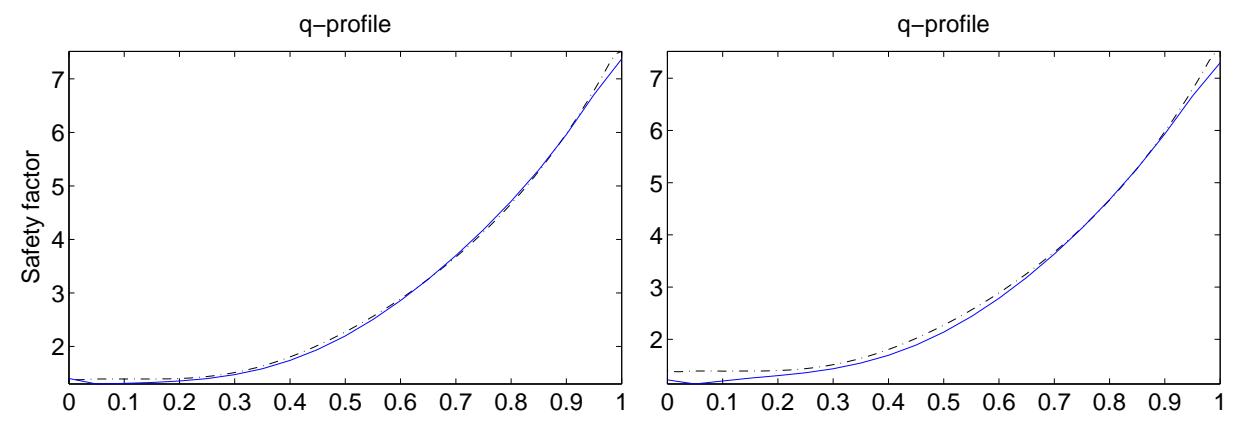

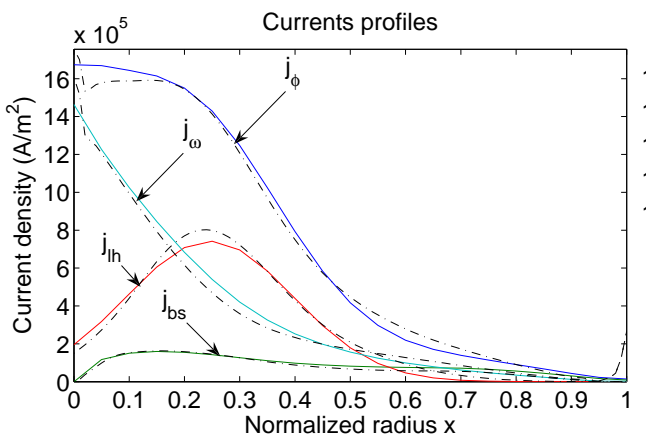

(a) With measured $T_{e}$ profile

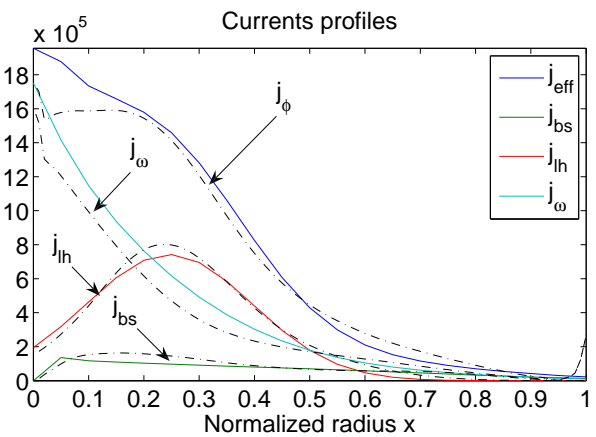

(b) With estimated $T_{e}$ profile

Figure 7. Comparison of $\psi_{\text {sim }}(-)$ with CRONOS (- - -) for shot TS-35109 at $t=7 \mathrm{~s}$, based on the safety factor profiles (top) and the current densities (effective $j_{\phi}, \mathrm{LH} j_{l h}$, ohmic $j_{\omega}$ and bootstrap $j_{b s}$ ) profiles (bottom).

illustrated in Figure 8(b).

To conclude on this comparison between $\psi_{\text {sim }}$ and experimental results or CRONOS, the proposed model provide for some satisfactory results (accurate enough for control applications), even with a long sampling time and a small number of discretisation points. The effects of the main control inputs are well represented and the computation time is small ( $5 s$ for shot TS-35109). Comparing the errors on the safety factor profiles introduced by the use of the temperature scaling laws show that the model, at least for the $q$-profile, is not too much sensible to these laws, as long as the orders of magnitude are suitably represented. For the magnetic measurement $\beta_{\theta}+l_{i} / 2$, the scaling laws introduce a constant bias.

\section{Conclusions}

We proposed in this work a new, control-oriented, model of the current diffusion in tokamak plasma. The current profile dynamics was modelled by the $1 D$ magnetic flux diffusion equation, using approximate formulae of the neoclassical resistivity coefficient and the bootstrap current. The non-inductive current sources were considered as Gaussian distributions depending on the control inputs, which are derived either from approximate theoretical formulae for the ECCD or from experimental scaling laws specifically developed from Hard X-ray Tore Supra data for the LHCD term (the proposed method is detailed and can easily be applied to other tokamaks, provided that Hard X-ray measurements are available). 

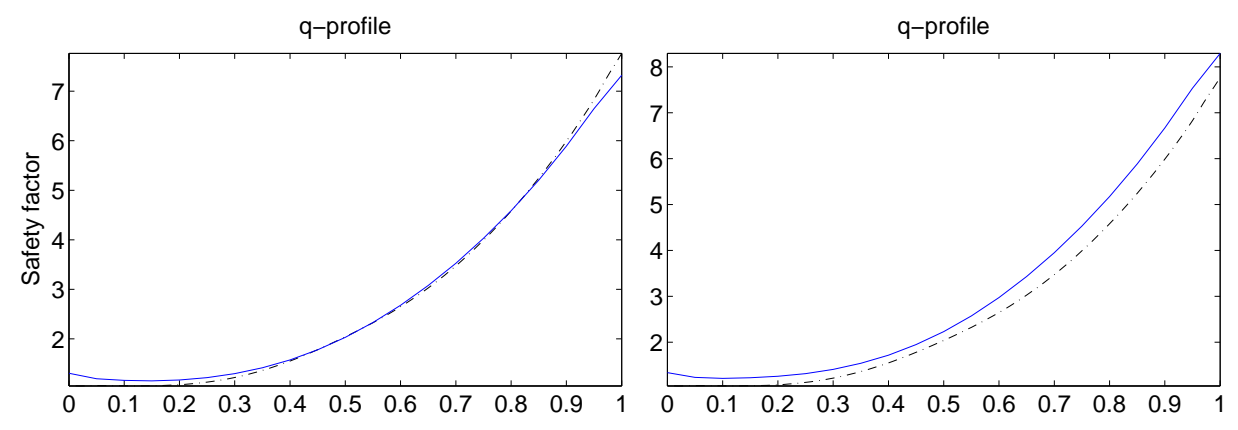

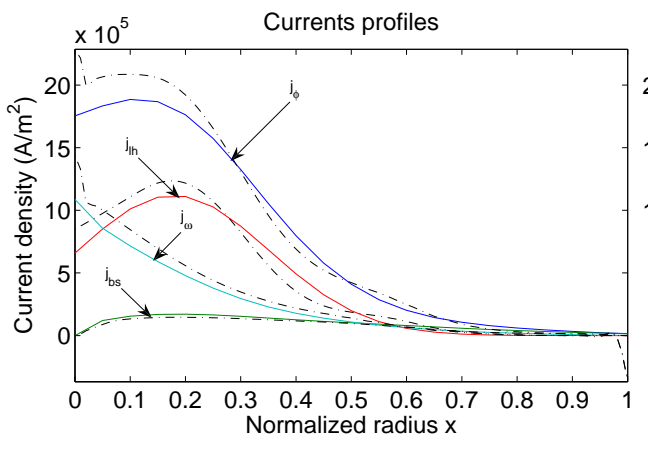

(a) With measured $T_{e}$ profile

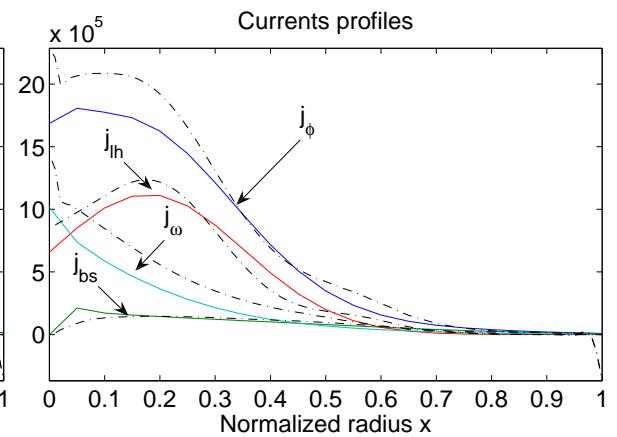

(b) With estimated $T_{e}$ profile

Figure 8. Comparison of $\psi_{\text {sim }}$ using $V_{\text {loop }}$ as a boundary condition (-) with CRONOS (- - -) for shot TS-35109 at $t=28.2 \mathrm{~s}$, based on the safety factor profiles (top) and the current densities (effective $j_{\phi}$, LH $j_{l h}$, ohmic $j_{\omega}$ and bootstrap $j_{b s}$ ) profiles (bottom).

The input-output relationships and the discretisation issues (in time and space) were detailed to provide for a model that is computationally efficient and robust, and particularly suited for advanced control of the plasma profiles. For example, it can be used directly to estimate the plasma evolution over a short time-range from real-time measurements and included in model-based predictive control schemes. The model structure also suggests that nonlinear analysis may be an interesting tool for further developments in plasma profile control and to estimate the associated performance limitation.

A new simulation tool was developed according to this model, to allow for a comparison with Tore Supra experimental data and CRONOS code outputs. Based on two different shots, this comparison was carried out on the magnetic measurements as well as the safety factor and current densities profiles. The precision of the model and its computational efficiency have shown to be particularly satisfying for future control applications. Such a tool can also estimate the safety factor, current densities, toroidal voltage and confinement efficiency in real time from the global parameters and temperature profiles measurements.

\section{Appendix A. Discretisation method}

This section presents a discretisation method that has a variable step in the spatial domain and is implicit-explicit in the temporal domain. The spatial discretisation method is motivated by the fact that an improved resolution may be desired in specific regions (such as the plasma 
centre) with a limited number of discretisation points. The choice of these points can be determined by the actuators/sensors placement or by the control objectives. The temporal discretisation method allows for a trade-off between numerical stability and precision. We consider the function $f(x, t)$ and denote by $f_{i, j}$ its value at $x_{i}, i=1, \ldots, N$, and at time $j \delta t$, where $\delta t$ is the sampling time and $j \in \mathbb{N}^{+}$is the time index considered.

\section{Appendix A.1. Variable-step spatial differentiation}

The sampling interval is defined as $\delta x_{i}=x_{i}-x_{i-1}$, with $\delta x_{1}=x_{1}=0$. Using Taylor's series, we can write the general formula

$$
f(x+\Delta x, t)=f(x, t)+\Delta x f^{\prime}(x, t)+\frac{\Delta x^{2}}{2} f^{\prime \prime}(x, t)+\mathrm{O}(3)
$$

where $f^{\prime}(\cdot) \doteq \partial f(\cdot) / \partial x$ and $\mathrm{O}(3)$ denotes $3^{r d}$ and higher order terms. At time $j \delta t$, we have that

$$
\begin{aligned}
& f_{i+1, j}=f_{i, j}+\delta x_{i+1} f^{\prime}\left(x_{i}, t\right)+\frac{\delta x_{i+1}^{2}}{2} f^{\prime \prime}\left(x_{i}, t\right)+\mathrm{O}(3) \\
& f_{i-1, j}=f_{i, j}-\delta x_{i} f^{\prime}\left(x_{i}, t\right)+\frac{\delta x_{i}^{2}}{2} f^{\prime \prime}\left(x_{i}, t\right)+\mathrm{O}(3) .
\end{aligned}
$$

The first order spatial derivative of $f(\cdot)$ is then computed by subtracting $f_{i+1, j}$ from $f_{i-1, j}$, which gives

$$
f^{\prime}\left(x_{i}, t\right)=\frac{f_{i+1, j}-f_{i-1, j}}{\delta x_{i+1}+\delta x_{i}}+\mathrm{O}(1) .
$$

Similarly, the second order derivative is obtained by adding $f_{i+1, j}$ and $f_{i-1, j}$ :

$$
f^{\prime \prime}\left(x_{i}, t\right)=2 \frac{f_{i+1, j}-2 f_{i}+f_{i-1, j}-\left(\delta x_{i+1}-\delta x_{i}\right) f^{\prime}\left(x_{i}, t\right)}{\delta x_{i+1}^{2}+\delta x_{i}^{2}}+\mathrm{O}(1) .
$$

Neglecting $\mathrm{O}(1)$, we finally write

$$
\begin{aligned}
& f^{\prime}\left(x_{i}, t\right)=d_{1}\left(f_{i+1, j}-f_{i-1, j}\right) \\
& f^{\prime \prime}\left(x_{i}, t\right)=d_{2} f_{i+1, j}-d_{3} f_{i, j}+d_{4} f_{i-1, j}
\end{aligned}
$$

with

$$
\begin{aligned}
& d_{1}(i)=\frac{1}{\delta x_{i+1}+\delta x_{i}}, \quad d_{2}(i)=\frac{4 \delta x_{i}}{\left(\delta x_{i+1}^{2}+\delta x_{i}^{2}\right)\left(\delta x_{i+1}+\delta x_{i}\right)} \\
& d_{3}(i)=\frac{4}{\delta x_{i+1}^{2}+\delta x_{i}^{2}} \quad \text { and } \quad d_{4}(i)=\frac{4 \delta x_{i+1}}{\left(\delta x_{i+1}^{2}+\delta x_{i}^{2}\right)\left(\delta x_{i+1}+\delta x_{i}\right)} .
\end{aligned}
$$

Note that the same formulas hold if the discretisation grid is time-varying.

\section{Appendix A.2. Variable-step spatial integration}

The spatial integration of $f(x, t)$ is performed based on the principle of Simpson's rule, which is to find a polynomial that fits the curve in three points. Such a polynomial is taken in Lagrange basis and writes as

$$
P(x)=f_{i, j} \frac{x-x_{i+1}}{x_{i}-x_{i+1}} \frac{x-x_{i+2}}{x_{i}-x_{i+2}}+f_{i+1, j} \frac{x-x_{i}}{x_{i+1}-x_{i}} \frac{x-x_{i+2}}{x_{i+1}-x_{i+2}}+f_{i+2, j} \frac{x-x_{i}}{x_{i+2}-x_{i}} \frac{x-x_{i+1}}{x_{i+2}-x_{i+1}} .
$$


The integral between two points $x_{i}$ and $x_{i+2}$ is then

$$
\begin{array}{rl}
\int_{x_{i}}^{x_{i+2}} & f(x, t) d x \approx \int_{x_{i}}^{x_{i+2}} P(x) d x \\
& \approx \frac{\delta x_{i+2}+\delta x_{i+1}}{6}\left[\frac{2 \delta x_{i+1}-\delta x_{i+2}}{\delta x_{i+1}} f_{i, j}+\frac{\left(\delta x_{i+1}+\delta x_{i+2}\right)^{2}}{\delta x_{i+1} \delta x_{i+2}} f_{i+1, j}+\frac{2 \delta x_{i+2}-\delta x_{i+1}}{\delta x_{i+2}} f_{i+2, j}\right] .
\end{array}
$$

For the general case where the integration is carried out between the two locations $x_{m}$ and $x_{n}$,

$$
\int_{x_{m}}^{x_{n}} f(x, t) d x \approx \sum_{k=0}^{\alpha_{i n t}-1} \int_{x_{m+2 k}}^{x_{m+2 k+2}} P(x) d x+\beta_{i n t} \frac{\delta x_{n}}{\delta x_{n}+\delta x_{n+1}} \int_{x_{n-1}}^{x_{n+1}} P(x) d x
$$

where

$$
\begin{cases}\alpha_{i n t}=(n-m) / 2 \quad \text { and } \quad \beta_{\text {int }}=0 & \text { if }(n-m) \text { is even, } \\ \alpha_{\text {int }}=(n-m-1) / 2 \quad \text { and } \quad \beta_{\text {int }}=1 & \text { if }(n-m) \text { is odd. }\end{cases}
$$

This integration method is particularly computationally efficient and useful from a control point of view since it allows to approximate the integral operator with a linear operator as

$$
\int_{0}^{x_{i}} f(x, t) d t \approx M_{i n t}(i, 1: i)\left[\begin{array}{c}
f_{1, j} \\
\vdots \\
f_{i, j}
\end{array}\right]
$$

where $M_{\text {int }} \in \mathbb{R}^{N \times N}$ is the integration matrix, constructed with the previous equations, and $M_{\text {int }}(i, 1: i)$ denotes the columns 1 to $i$ of row $i$.

\section{Appendix A.3. Temporal differentiation}

It is performed using an implicit-explicit scheme, as proposed in [33]. For the general case where the function to be discretised writes as $\dot{f}(x, t)=g(x, t)$, the time derivative $\dot{f}(\cdot)$ is obtained with

$$
\left(\frac{f_{i, j+1}-f_{i, j}}{\delta t}\right)=h\left(\frac{f_{i, j+1}-f_{i, j}}{\delta t}\right)_{e x}+(1-h)\left(\frac{f_{i, j+1}-f_{i, j}}{\delta t}\right)_{i m}
$$

where $h \in[0,1]$ and

$$
\left(\frac{f_{i, j+1}-f_{i, j}}{\delta t}\right)_{e x}=g_{i, j}, \quad\left(\frac{f_{i, j+1}-f_{i, j}}{\delta t}\right)_{i m}=g_{i, j+1} .
$$

Note that for the special case of the diffusion equation, $g$ typically depends on $f^{\prime}, f^{\prime \prime}$ and the source terms. The temporal discretisation method described here corresponds to the CrankNicholson scheme if $h=0.5$.

\section{Appendix B. Gaussian distribution}

The ECCD and LHCD systems generate distributed plasma current deposits, which are represented as Gaussian distribution. The general equation describing such a distribution is given by

$$
j_{n i}^{\prime}(x, t)=j_{n i}(x, t) \frac{\mu(t)-x}{\sigma(t)}
$$


where, in the classical probabilistic terminology, $\mu(t)$ is the mean and $\sigma(t)$ is the variance of the function considered. Integrating the previous equation, we have

$$
j_{n i}(x, t)=\vartheta(t) e^{-(\mu(t)-x)^{2} / 2 \sigma(t)}
$$

where $\vartheta(t)$ sets the maximum value of $j_{n i}(x, t)$ (at $x=\mu$ ). A discretised version is obtained thanks to the central difference method as

$$
\frac{j_{n i, i+1, j}-j_{n i, i-1, j}}{\delta x_{i+1}+\delta x_{i}}=j_{n i, i, j} \frac{\mu_{j}-x_{i}}{\sigma_{j}} \Leftrightarrow j_{n i, i+1, j}=\left(\delta x_{i+1}+\delta x_{i}\right) \frac{\mu_{j}-x_{i}}{\sigma_{j}} j_{n i, i, j}+j_{n i, i-1, j}
$$

with the boundary conditions

$$
j_{n i, 1, j}=\vartheta_{j} e^{-\mu_{j}^{2} / 2 \sigma_{j}} \quad \text { and } \quad j_{n i, 2, j}=\vartheta_{j} e^{-\left(\mu_{j}-x_{2}\right)^{2} / 2 \sigma_{j}}
$$

for both CD systems.

\section{Appendix C. Optimal fitting method}

Given a set of experimental measurements and an appropriate fitting function, this section describes an optimal identification method to determine the fitting function parameters. Some proper references on numerical solutions by iterative search and gradient methods can be found in [57]. More specifically, a similar design method was used in [58] for the optimal control of systems with stochastic components in the dynamics. We consider here the steady state (time-invariant) behaviour of a physical quantity as a function described by a set of $n_{m}$ measurements (i.e. the sampling times), which has to be large enough to ensure that the resulting fitting function represents the physical phenomena. The set of measured inputs is

$$
\mathscr{I}=\left\{I_{1}, I_{2}, \ldots, I_{n_{i}}\right\} \in \mathbb{R}^{n_{m} \times n_{i}}
$$

where $n_{i}$ is the number of physical quantities that influence the identified one, and the measured outputs are denoted by $y \in \mathbb{R}^{n_{m}}$. The estimated output is then

$$
\hat{y}(\vartheta, i)=f(\mathscr{I}(i), \vartheta), \quad i=1 \ldots n_{m}
$$

where $f(\cdot)$ is the fitting law, $\mathscr{I}(i)$ corresponds to the input measurements of the $i^{\text {th }}$ experiment and $\vartheta$ is the vector of design parameters.

\section{Appendix C.1. General formulation}

A classical identification problem is to find $\vartheta$ that minimizes the difference between the measured and estimated data. This is done in this section by choosing a cost function $J$ which reflects the variance of the estimation error from a given set of measurements:

$$
J(\vartheta)=\frac{1}{n_{m}} \sum_{i=1}^{n_{m}}\|y(i)-\hat{y}(\vartheta, i)\|^{2}
$$


The output error is then minimized for $\vartheta^{*}$ satisfying

$$
\vartheta^{*}=\arg \min _{\vartheta} J(\vartheta)
$$

This optimization problem can be solved with a descent algorithm, using the sensitivity of $\hat{y}(i)$ with respect to $\vartheta$, expressed as $S(\vartheta, i) \doteq \partial \hat{y}(i) / \partial \vartheta$. The gradient writes as

$$
\nabla J(\vartheta)=-\frac{2}{n_{m}} \sum_{i=1}^{n_{m}}(y(i)-\hat{y}(\vartheta, i)) S(\vartheta, i)
$$

and the optimal parameter $\vartheta^{*}$ is obtained by moving along the steepest slope $-\nabla J(\vartheta)$ with a step $\alpha$, which has to be small enough to ensure that $\dot{\vartheta}=-\alpha \nabla J(\vartheta)$ converges to $\vartheta^{*}$. This step is chosen according to Newton's method and writes as $\alpha \doteq(\Psi J(\vartheta)+v I)^{-1}$, where $v$ is a positive constant introduced to ensure strict positiveness and $\Psi J(\vartheta)$ is the pseudo-Hessian, derived using the Gauss-Newton approximation as

$$
\Psi J(\vartheta)=\frac{2}{n_{m}} \sum_{i=1}^{n_{m}} S(\vartheta, i) S(\vartheta, i)^{T}
$$

The optimal set of fitting parameters is finally obtained with the variation law

$$
\begin{aligned}
& \vartheta_{l+1}=\vartheta_{l}-\alpha_{l} \nabla J\left(\vartheta_{l}\right) \\
& \alpha_{l}=\left(\Psi J\left(\vartheta_{l}\right)+v I\right)^{-1}
\end{aligned}
$$

for $l$ sufficiently large.

Remark 4 The convergence speed of the algorithm is inversely proportional to the design parameter $v$ but choosing this parameter too small may create some oscillations in the algorithm.

\section{Appendix C.2. Linear regression}

The previous method is particularly efficient when the output is estimated with a linear regression technique. Indeed, in that case we can write $\hat{y}(\vartheta, i)=f(\mathscr{I}(i)) \times \vartheta, i=1 \ldots n_{m}$, and the sensitivity function is simplified as

$$
S(\vartheta, i)=S(i)=f(\mathscr{I}(i))
$$

Consequently, the pseudo-Hessian function $\Psi J$ and $\alpha$ do not depend on the design parameters. A commonly used fitting function is given by

$$
\hat{O}=\alpha_{0} I_{1}^{\alpha_{1}} I_{2}^{\alpha_{2}} I_{3}^{\alpha_{3}} \ldots I_{n_{i}}^{\alpha_{n_{i}}}
$$

The linear regression is then performed with the output

$$
\left\{\begin{array}{l}
y=\ln (O) \\
\hat{y}(\vartheta)=\ln \left(\alpha_{0}\right)+\alpha_{1} \ln \left(I_{1}\right)+\alpha_{2} \ln \left(I_{2}\right)+\ldots+\alpha_{n_{i}} \ln \left(I_{n_{i}}\right)
\end{array}\right.
$$

where $O$ is the measured output, and the previous algorithm is applied with

$$
\begin{aligned}
\alpha & =\left[\begin{array}{lllll}
\ln \left(\alpha_{0}\right) & \alpha_{1} & \alpha_{2} & \ldots & \alpha_{n_{i}}
\end{array}\right]^{T} \\
f(\mathscr{I}) & =\left[\begin{array}{lllll}
1_{n_{m}} & \ln \left(I_{1}\right) & \ln \left(I_{2}\right) & \ldots & \ln \left(I_{n_{i}}\right)
\end{array}\right]
\end{aligned}
$$

where $1_{n_{m}}$ is a column vector of $n_{m}$ ones. 


\section{Appendix C.3. LH deposit estimation}

The proposed method is applied to the estimation of the lower hybrid current deposit width $W_{x}$ with the fitting function (with 6 parameters)

$$
\hat{w}_{h x r}=\alpha_{0} B_{\phi_{0}}^{\alpha_{1}} I_{p}^{\alpha_{2}} \bar{n}^{\alpha_{3}} P_{L H}^{\alpha_{4}} N_{/}^{\alpha_{5}}
$$

The optimal parameter vector is computed from the variation law (C.1)-(C.2) with the sensitivity function (C.3)-(C.4). A set of 111 averaged measurements, performed on Tore Supra shots ranging between the shot numbers 34496 and 36165, determines the values of $\mathscr{I}$ and $O$. This optimal parameter determination method is also applied to estimate the centre of the deposit profile (radial position of the maximum value) and provide for the coefficients $\alpha_{0}, \ldots, \alpha_{5}$ of the relationship

$$
\hat{\mu}_{h x r}=\alpha_{0}^{\prime} B_{\phi_{0}}^{\alpha_{1}^{\prime}} I_{p}^{\alpha_{2}^{\prime}} \bar{n}_{3}^{\alpha_{3}^{\prime}} P_{L H}^{\alpha_{4}^{\prime}} N_{/ /}^{\alpha_{5}^{\prime}}
$$

\section{Acknowledgments}

The authors would like to thank Jean-Pierre Quadrat, François Delebecque, Jacques Blum and François Gallère for the interesting discussions on different aspects of this model. We are also most grateful to the technical support of Tore Supra team, to the invaluable contributions of the Associations involved in this project and to the constructive insights of the reviewers. This work has been supported in parts by the European Fusion Development Agreement (EFDA) and by the European Commission through the projects SOCRADES and HYCON.

\section{References}

[1] Taylor T S. Physics of advanced tokamaks. Plasma Phys. Control. Fusion, 39(B):47-73, December 1997.

[2] Joffrin E et al. Integrated scenario in JET using real-time profile control. Plasma Phys. Control. Fusion, 45:A367-A383, 2003.

[3] Gormezano C. High performance tokamak operation regimes. Plasma. Phys. Control. Fusion, 41(12B):B367-B380, December 1999.

[4] Wolf R C. Internal transport barriers in tokamak plasmas. Plasma. Phys. Control. Fusion, 45(1):R1-R91, January 2003.

[5] Jaun A, Fasoli A, Vaclavik J, and Villard L. Stability of Alfvén eigenmodes in optimized tokamaks. Nucl. Fus., 40(7):1343-1348, July 2000.

[6] Wijnands T, Van Houtte D, Martin G, Litaudon X, and Froissard P. Feedback control of the current profile on Tore Supra. Nucl. Fus., 37(6):777-791, June 1997.

[7] Synakowski E J et al. Roles of electric field shear and shafranov shift in sustaining high confinement in enhanced reversed shear plasmas on the TFTR tokamak. Phys. Rev. Lett., 78(15):2972-2975, April 1997.

[8] Ide S, Fujita T, Naito O, and Seki M. Sustainment and modification of reversed magnetic shear by LHCD on JT-60U. Plasma. Phys. Control. Fusion, 38(10):1645-1652, October 1996.

[9] Sakamoto Y et al. Characteristics of internal transport barriers in JT-60U reversed shear plasmas. Nucl. Fus., 41(7):865-872, July 2001.

[10] Ide S, Fujita T, Suzuki T, Naito O, Kamada Y, and Seki M. Combined non-inductive current drive in a high confinement reversed magnetic shear plasma at high normalized density in JT-60U. Plasma. Phys. Control. Fusion, 44(11):L63-L69, November 2002. 
[11] Wade M R et al. Achieving and sustaining steady state advanced tokamak conditions on DIII-D. In Proc. $19^{\text {th }}$ Fusion Energy Conf. (IAEA-CN-94), pages EX-P3/16, Lyon, France, October 2002.

[12] Rice J E et al. Pressure profile control of internal transport barrier plasmas in Alcator C-Mod. Nucl. Fusion, 43:781788, 2003.

[13] F. Crisanti et al. JET quasistationary internal-transport-barrier operation with active control of the pressure profile. Phys. Rev. Lett., 88(14):145004:1-4, March 2002.

[14] Litaudon X et al. Towards fully non-inductive current drive operation in JET. Plasma. Phys. Control. Fusion, 44(7):1057-1086, July 2002.

[15] Litaudon X et al. Progress towards steady-state operation and real-time control of internal transport barriers in JET. Nucl. Fus., 43(7):565-572, July 2003.

[16] Mazon D et al. Real-time control of internal transport barriers in JET. Plasma. Phys. Control. Fusion, 44(7):1087-1104, July 2002.

[17] Mazon D et al. Real time control of the current profile in JET. In 29th EPS Conf. on Plasma Phys. Control. Fusion, Montreux, Switzerland, June 2002.

[18] Mazon D et al. Active control of the current density profile in JET. Plasma. Phys. Control. Fusion, 45(7):L47-L54, 2003.

[19] Lyapunov A M. The general problem of the stability of motion (in Russian). Taylor \& Francis (English translation, 1992), 1892.

[20] Kokotovi P and Arcak M. Constructive nonlinear control: a historical perspective. Automatica, 37(5):637662, May 2001.

[21] Findeisen R, Imsland L, Allgöwer F, and Foss B A. State and output feedback nonlinear model predictive control: An overview. Europ. J. Contr., 9(2-3):190-207, 2003.

[22] Vidyasagar M. Nonlinear Systems Analysis. Prentice-Hall, Englewood Cliffs, NJ, 1992.

[23] Khalil H K. Nonlinear System, Second Edition. Prentice Hall, Upper Saddle River, NJ, 1996.

[24] Sastry S. Nonlinear Systems: Analysis, Stability, and Control. Spring-Verlag, New York, 1999.

[25] Billings S A, Gray J O, and Owens D H, editors. Nonlinear system design. Peter Peregrinus Ltd, London, UK, 1984.

[26] Slotine J-J and Li W. Applied nonlinear control. Prentice Hall, Englewood Cliffs, New Jersey, 1991.

[27] Nijmeijer H and van der Schaft A. Nonlinear dynamical control systems. Springer-Verlag, New York, 1990.

[28] Isidori A. Nonlinear Control Systems. Springer-Verlag, New York, 1995.

[29] Mohler R. Nonlinear Systems. Prentice-Hall, Englewood Cliffs, NJ, 1991.

[30] Krstic M, Kannellakopoulos I, and Kokotovic P. Nonlinear and Adaptive Control Systems Design. Wiley, New York, 1995.

[31] Freeman R A and Kokotović P V. Robust nonlinear control design: state-space and Lyapunov Techniques. Birkhäuser, Boston, 1996.

[32] Blum J. Numerical simulation and optimal control in plasma physics with applications to Tokamaks. Modern Applied Mathematics. Wiley / Gauthier-Villars, 1989.

[33] Brégeon R, Joffrin E, and Litaudon X. Résolution de l'équation de diffusion du flux et de la chaleur en coordonnées toroidales. Association EUR-CEA / DRFC / Tore Supra, NT $\Phi$ 123, July 1997. Private communication.

[34] Brégeon R. Évolution résistive du profil de courant dans les tokamaks, application à l'optimisation des décharges de Tore Supra. PhD thesis, Université de Provence (Aix-Marseille I), CEA - Cadarache, France, November 1998.

[35] Clémençon A, Guivarch C, Eury S P, Zou X L, and Giruzzi G. Analytical solution of the diffusion equation in a cylindrical medium with step-like diffusivity. Physics of Plasmas, 11(11):4998-5009, 2004.

[36] Erba M, Aniel T, Basiuk V, Becoulet A, and Litaudon X. Validation of a new mixed Bohm/gyro-Bohm model for electron and ion heat transport against the ITER, Tore Supra and START database discharges. Nucl. Fusion, 38(7):1013-1028, July 1998.

[37] Witrant E. Parameter dependant identification of nonlinear distributed systems: Application to tokamak plasmas. Technical report TRITA-EE 2007:029, Royal Institute of Technology (KTH), Stockholm, 
Sweden, June 2007.

[38] ITER Physics Expert Groups. Chapter 2: Plasma confinement and transport. Nucl. Fusion, 39(12):21752249, 1999.

[39] Hirshman S P, Hawryluk R J, and Birge B. Neoclassical conductivity of a tokamak plasma. Nucl. Fusion, 17(3):611-614, 1977.

[40] Wesson J. Tokamaks. Number 48 in Oxford engineering science. Oxford Science Publications, New York, $2^{\text {nd }}$ edition, 1997.

[41] Joffrin E, Basiuk V, Bregeon R, and Litaudon X. Determination of non-inductive current profiles in Tore Supra. Plasma Phys. Control. Fusion, 42:1227-1239, 2000.

[42] Hirshman S P. Finite-aspect-ratio effects on the bootstrap current in tokamaks. Phys. of Fluids, 31(10):3150-3152, October 1988.

[43] Kessel C E. Bootstrap current in a tokamak. Nucl. Fusion, 34(9):1221-1238, 1994.

[44] Hoang G T and Budny R V. The bootstrap fraction in TFTR. In AIP Conference Proceedings, volume 403:1, pages 235-238, 1997.

[45] Huang Y and McColl W F. Analytical inversion of general tridiagonal matrices. J. Phys. A: Math. Gen., 30:7919-7933, 1997.

[46] Kazarian-Vibert F, Litaudon X, Moreau D, Arslanbekov R, Hoang G T, and Peysson Y. Full steady-state operation in Tore Supra. Plasma Phys. Control. Fusion, 38:2113-2131, 1996.

[47] Fisch N J. Theory of current drive in plasmas. Reviews of Modern Physics, 59(1):175-234, January 1987.

[48] Giruzzi G. Impact of electron trapping on RF current drive in tokamaks. Nucl. Fusion, 27(11):1934-1939, 1987.

[49] Giruzzi G, Ségui J-L, Pecquet A-L, and Gil C. Observation of the $m=1$ mode by microwave transmission measurements in Tore Supra. Nucl. Fusion, 31(11):2158-2162, 1991.

[50] Imbeaux F. Etude de la propagation et de l'absorption de l'onde hybride dans un plasma de tokamak par tomographie X haute énergie. PhD thesis, Université Paris XI Orsay, Orsay, France, September 1999.

[51] Barana O, Mazon D, Caulier G, Garnier D, Jouve M, Laborde L, and Peysson Y. Real-time determination of suprathermal electron local emission profile from Hard X-ray Measurements on Tore Supra. IEEE Trans. on Nuclear Science, 53:1051-1055, 2006.

[52] Goniche M et al. Lower Hybrid current drive efficiency on Tore Supra and JET. In $16^{\text {th }}$ Topical Conference on Radio Frequency Power in Plasmas, Parkcity, USA, June 2005.

[53] Hoang G T, Lecoustey P, and Moreau D. Mesures dia/paramagnétiques de tore supra. Private communication PHY/NTT-1991.035, Ass. EUR-CEA/DRFC, 1991.

[54] Basiuk V et al. Simulations of steady-state scenarios for Tore Supra using the CRONOS code. Nucl. Fusion, 43:822-830, 2003.

[55] Liu Y and Jacobsen E W. Grey-box modelling of distributed parameter processes - application to chromatography. In Proceedings of 14th European Symposium on Computer Aided Process Engineering, Porto, Portugal, May 2004.

[56] Liu Y. Grey-box identification of distributed parameter systems. PhD thesis, KTH Electrical Engineering, Stockholm, Sweden, May 2005.

[57] Ljung L. System Identification: Theory for the User. Information and System Sciences. PTR Prentice Hall, Upper Saddle River, NJ, $2^{\text {nd }}$ edition, 1999.

[58] Witrant E, Georges D, and Canudas de Wit C. Optimal control design for the stabilization of network controlled systems. In Proc. of the IEEE American Control Conf., Minneapolis, MN (USA), June 2006. 